\title{
The self-consistent description of stellar equilibrium with axial rotation
}

\author{
Vavrukh M. V., Tyshko N. L., Dzikovskyi D. V., Stelmakh O. M. \\ Ivan Franko National University of Lviv, \\ 8 Kyrylo and Methodiy Str., 79005, Lviv, Ukraine
}

(Received 9 July 2019; Accepted 1 October 2019)

\begin{abstract}
New method for the description of mechanical equilibrium of stellar structure with axial rotation was proposed. The self-consistent calculation is based on simultaneous use of differential and integral forms of mechanical equilibrium equation, which allows us to correctly determine the integration constants. In the frame of polytropic model with indexes $n=0$ and 1 were first obtained the analytical solutions, for $n=2$ and 3 numerically. The geometrical parameters of stellar surface as well as mass, volume and moment of inertia were calculated as the functions of angular velocity. It was found the maximal value of angular velocity in which the stability is disturbed. Obtained results improve the results of E. Milne, S. Chandrasekhar and R. James, obtained with help of the approximate numerical integration of mechanical equilibrium equation.
\end{abstract}

Keywords: polytropic stars, heterogeneous ellipsoids, axial rotation, mechanical equilibrium equation, stability of stars.

2000 MSC: $85-08,85 \mathrm{~A} 15$

UDC: 524.3

DOI: $10.23939 / \mathrm{mmc} 2019.02 .153$

\section{Introduction}

In the late XIX-th - early XX-th centuries the works of H. Lane [1], K. Emden [2], R. Fowler [3], A. Ritter [4], W. Kelvin [5], A. Eddington [6] and other researchers laid the foundations of the theory of internal stellar structure. At the first stage it was only considered the mechanical equilibrium of normal stars - the equilibrium of gravity and internal pressure of the spherical symmetry models which did not take into account the energy sources of stars, unknown at that time. The result of this stage was the K. Emden's book [2] of 1907. In the late works of E. Milne [7], S. Chandrasekhar [8], R. James [9] and other authors, which were published in the first half of the XX-th century, this theory was generalized to the case of polytropic models with solid axial rotation. The discovery of thermonuclear reactions solved the problem of stars energy sources. The modern theory of normal stars generalizes the polytropic theory and is based on the system of the internal structure equations, which take into account both the mechanical and thermal equilibrium of these objects.

However, the polytropic theory has not lost its significance now, because its mathematical apparatus easily adapts for the description of internal structure of compact objects, in particular the degenerate dwarfs. After all, the equation of state of electron-nuclear model with relativistic electron subsystem has asymptotically (at high and low densities of matter) polytropic character. Because of that the solutions of equilibrium equations for the polytropic stars (with or without rotation) play the role of zero approximation for the solutions of equilibrium equation of degenerate dwarfs [10]. The axial rotation of non-magnetic dwarfs is the only reason which causes an increase of their mass. Therefore, one of the topical problems of degenerate dwarfs' theory is the calculation of maximal mass and setting the limit of stability with respect to the angular velocity. Although this problem was investigated both through numerical integration of equilibrium equations and with help of the variational method [11], still the problem is far from the final solution. 
In accordance with above said the actual task is to find the mechanical equilibrium solutions of polytropic stars in wide range for the angular velocity and determining the limits of stability on this basis. Usually one uses the differential equations of mechanical equilibrium. In our work they are supplemented by the integral form of these equations, which makes it possible to strictly define the integration constants. It also provides a direct way of finding the dependence of the equilibrium equations and macroscopic characteristics of star on the angular velocity.

\section{General relations}

In the polytropic theory of stars the equation of state is modeled by the dependence [12]

$$
P(\mathbf{r})=K \rho^{\nu}(\mathbf{r}) \equiv K \rho^{1+1 / n}(\mathbf{r}),
$$

where $P(\mathbf{r})$ is the pressure in point with radius-vector $\mathbf{r}, \rho(\mathbf{r})$ is the local density of matter, $K$ and $\nu$ are the constants. In general case (in the presence of rotation) the equilibrium equation is rewritten in non-inertial (rotating) coordinate system in the form

$$
\nabla P(\mathbf{r})=-\rho(\mathbf{r})\left\{\nabla \Phi_{\text {grav }}(\mathbf{r})+\nabla \Phi_{c}(\mathbf{r})\right\}
$$

where

$$
\Phi_{\text {grav }}(\mathbf{r})=-G \int \frac{d \mathbf{r}^{\prime} \rho\left(\mathbf{r}^{\prime}\right)}{\left|\mathbf{r}-\mathbf{r}^{\prime}\right|}
$$

is the gravitational potential inside the star and $\Phi_{c}(\mathbf{r})$ is the centrifugal potential. If the axis $O z$ of spherical coordinate system coincides with the axis of rotation then

$$
\Phi_{c}(\mathbf{r})=-\frac{1}{2} \omega^{2} r^{2} \sin ^{2} \theta
$$

Here $\theta$ is the polar angle, $\omega$ is the angular velocity of reference frame, which is considered a constant.

Substituting the expressions (1), (3) and (4) in the equation (2) and using the identity

$$
\left(1+\frac{1}{n}\right) \rho^{1 / n-1}(\mathbf{r}) \nabla \rho(\mathbf{r})=(1+n) \nabla \rho^{1 / n}(\mathbf{r}),
$$

the equilibrium equation is obtained in the form of differential equation that determines the density distribution,

$$
K(1+n) \Delta \rho^{1 / n}(\mathbf{r})=-4 \pi G \rho(\mathbf{r})+\frac{1}{2} \omega^{2} \Delta\left(r^{2} \sin ^{2} \theta\right) .
$$

In the presence of axial symmetry $(\rho(\mathbf{r})=\rho(r, \theta))$ the Laplace operator is written in the form

$$
\Delta=\Delta_{r}+\frac{1}{r^{2}} \Delta_{\theta}, \quad \Delta_{r}=\frac{1}{r^{2}} \frac{\partial}{\partial r}\left(r^{2} \frac{\partial}{\partial r}\right), \quad \Delta_{\theta}=\frac{\partial}{\partial t}\left(1-t^{2}\right) \frac{\partial}{\partial t}
$$

at $t=\cos \theta$, therefore $\Delta\left(r^{2} \sin ^{2} \theta\right)=4$. Introducing the dimensionless radial coordinate $\xi=r / \lambda_{n}$, as well as using the substitution

$$
\rho(r, \theta)=\rho_{c} Y^{n}(\xi, \theta)
$$

where $\rho_{c}$ is the density of matter in stellar center, we transform the equation (5) to the dimensionless form

$$
\Delta(\xi, \theta) Y(\xi, \theta)=\Omega^{2}-Y^{n}(\xi, \theta) .
$$

Herewith the scale $\lambda_{n}$, dimensionless angular velocity $\Omega$ and Laplacian are determined by the relations

$$
K(1+n)=4 \pi G \lambda_{n}^{2} \rho_{c}^{1-1 / n}, \quad \Omega^{2}=\omega^{2}\left(2 \pi G \rho_{c}\right)^{-1},
$$

Mathematical Modeling and Computing, Vol. 6, No. 2, pp. 153-172 (2019) 


$$
\Delta(\xi, \theta)=\Delta_{\xi}+\frac{1}{\xi^{2}} \Delta_{\theta}, \quad \Delta_{\xi}=\frac{1}{\xi^{2}} \frac{\partial}{\partial \xi}\left(\xi^{2} \frac{\partial}{\partial \xi}\right)
$$

According to the definition (6) $Y(0, \theta)=1$ and the condition $\partial Y(\xi, \theta) / \partial \xi=0$ at $\xi=0$ corresponds to the solutions, regular in the vicinity $\xi=0$. At the large values $\Omega$ it is possible non-monotonic dependence $Y(\xi, \theta)$ on the variable $\xi$ in the equator region and leakage of matter. The stability conditions of stars in the equator region

$$
Y\left(\xi, \frac{\pi}{2}\right)=0, \quad \frac{\partial}{\partial \xi} Y\left(\xi, \frac{\pi}{2}\right)=0
$$

determine the maximal legitimate value of the parameter $\Omega_{m a x}^{(n)}$ and the corresponding value of the equatorial radius $\xi_{e}^{\max }(n)$. According to the definition (6), physical meaning only have the positive solutions of equation (7), which is the two-dimensional differential equation of second order in partial derivatives with two dimensionless parameters $n, \Omega \geqslant 0$.

The equation (7) is similar to the Poisson equation, therefore it can formally be considered as the equation for dimensionless gravitational potential, which is created by the dimensionless density distribution $(4 \pi)^{-1} \cdot\left\{\Omega^{2}-Y^{n}(\xi, \theta)\right\}$. In this regard, this equation can be rewritten in the integral form

$$
Y(\xi, \theta)=1+\sum_{l=1}^{\infty} C_{2 l} \xi^{2 l} P_{2 l}(t)-\frac{1}{4 \pi} \int\left\{\Omega^{2}-Y^{n}\left(\xi^{\prime}, \theta^{\prime}\right)\right\} Q\left(\boldsymbol{\xi}, \boldsymbol{\xi}^{\prime}\right) d \boldsymbol{\xi}^{\prime},
$$

where $C_{2 l}$ are the integration constants, $P_{2 l}(t)$ is the Legendre's polynomial of $2 l$-th order, the kernel of the equation is

$$
Q\left(\boldsymbol{\xi}, \boldsymbol{\xi}^{\prime}\right)=\left\{\left|\boldsymbol{\xi}-\boldsymbol{\xi}^{\prime}\right|^{-1}-\left(\xi^{\prime}\right)^{-1}\right\},
$$

and the integration is performed over the stellar volume. Taking into account the identity

$$
\Delta(\xi, \theta)\left\{\xi^{l} P_{l}(t)\right\}=0,
$$

it is easy to verify that the equations (7) and (9) are equivalent.

The root of equation $Y\left(\xi_{0}(\theta), \theta\right)=0$ determines the equation of the second-order curve (which is close to the ellipse), the rotation of which relative to the axis $O z$ is forms stellar surface. The expressions for mass, volume and moment of inertia of a star are as follows:

$$
\begin{aligned}
M(n, \Omega) & =2 \pi \lambda_{n}^{3} \rho_{c} \int_{0}^{\pi} \sin \theta d \theta \int_{0}^{\xi_{0}(\theta)} \xi^{2} Y^{n}(\xi, \theta) d \xi, \quad V(n, \Omega)=\frac{2 \pi}{3} \lambda_{n}^{3} \int_{0}^{\pi} \sin \theta \xi_{0}^{3}(\theta) d \theta, \\
I(n, \Omega) & =4 \pi \lambda_{n}^{5} \rho_{c} \int_{0}^{\pi} \sin ^{3} \theta d \theta \int_{0}^{\xi_{0}(t)} \xi^{4} Y^{n}(\xi, \theta) d \xi .
\end{aligned}
$$

At the arbitrary value of the polytropic index $n$ the gravitational potential inside the star (3) is related to dimensionless potential

$$
\Phi_{n}(\boldsymbol{\xi})=-\frac{1}{4 \pi} \int \frac{Y^{n}\left(\boldsymbol{\xi}^{\prime}\right)}{\left|\boldsymbol{\xi}-\boldsymbol{\xi}^{\prime}\right|} d \boldsymbol{\xi}^{\prime}
$$

with such expression

$$
\Phi_{\text {grav }}(\mathbf{r})=4 \pi G \lambda_{n}^{2} \rho_{c} \Phi_{n}(\boldsymbol{\xi}) .
$$

Rewriting the equation (2) in dimensionless variables, we obtain the relation

$$
\frac{\partial}{\partial \xi}\left\{\Phi_{n}(\xi, \theta)+Y(\xi, \theta)\right\}=\frac{\Omega^{2}}{3} \xi\left\{1-P_{2}(t)\right\} .
$$


The equation (9) can be represented in terms $Y(\xi, \theta), \Phi_{n}(\xi, \theta)$, namely

$$
Y(\xi, \theta)+\left\{\Phi_{n}(\xi, \theta)-\Phi_{n}(0,0)\right\}=1+\sum_{l=1} C_{2 l} \xi^{2 l} P_{2 l}(t)+\Omega^{2}\left\{\Phi_{0}(\xi, \theta)-\Phi_{0}(0,0)\right\}
$$

where $\Phi_{0}(\xi, \theta)$ is the dimensionless model potential with the constant density $\rho_{0}=1$. This allows us to convert the equation (11) to such form

$$
\frac{\partial}{\partial \xi}\left\{\sum_{l=1} C_{2 l} \xi^{2 l} P_{2 l}(t)+\Omega^{2}\left[\Phi_{0}(\xi, \theta)-\Phi_{0}(0,0)\right]\right\}=\xi \frac{\Omega^{2}}{3}\left(1-P_{2}(t)\right) .
$$

The difference of potentials $\Phi_{0}(\xi, \theta)-\Phi_{0}(0,0)$ is easy to calculate using expansion in series of kernel $Q\left(\boldsymbol{\xi}, \boldsymbol{\xi}^{\prime}\right)$ by Legendre polynomials and performing integration over the variable $\boldsymbol{\xi}^{\prime}$ within rotational ellipsoid with eccentricity $e$ and equatorial radius $\xi_{e}$, which we will define in a self-consistent manner later. In this way we find that

$$
\Phi_{0}(\xi, \theta)-\Phi_{0}(0,0)=-\frac{1}{4 \pi} \int d \boldsymbol{\xi}^{\prime} Q\left(\boldsymbol{\xi}, \boldsymbol{\xi}^{\prime}\right)=\frac{\xi^{2}}{6}-\frac{\xi^{2}}{2} P_{2}(t) \int_{-1}^{+1} d t^{\prime} P_{2}\left(t^{\prime}\right) \int_{\xi}^{\xi_{0}\left(t^{\prime}\right)} \frac{d \xi^{\prime}}{\xi^{\prime}}
$$

where

$$
\xi_{0}\left(t^{\prime}\right)=\xi_{e}\left\{1+\left(t^{\prime}\right)^{2} \frac{e^{2}}{1-e^{2}}\right\}^{-1 / 2}
$$

Integrating over the variables $\xi^{\prime}$ and $t^{\prime}$ we obtain the final expression (see [11]):

$$
\begin{aligned}
& \Phi_{0}(\xi, \theta)-\Phi_{0}(0,0)=\frac{\xi^{2}}{6}+\frac{\xi^{2}}{2} P_{2}(t) I(e), \\
& I(e)=\frac{2}{3}+\frac{1-e^{2}}{e^{2}}-\frac{\left(1-e^{2}\right)^{1 / 2}}{e^{3}} \arcsin e .
\end{aligned}
$$

Varying the eccentricity in the limits $0 \leqslant e \leqslant 1$ the function $I(e)$ changes in the region $2 e^{2} / 15 \leqslant$ $I(e) \leqslant 2 / 3$. Substituting the expression (14) in the equation (12) we get the equality

$$
\sum_{l=1} C_{2 l} 2 l \xi^{2 l-2} P_{2 l}(t)=-\frac{\Omega^{2}}{3} P_{2}(t)(1+3 I(e)) .
$$

According to the orthogonality of the Legendre's polynomials it follows that

$$
C_{2}=-\frac{\Omega^{2}}{6}(1+3 I(e))
$$

and all others constants $C_{2 l}=0$.

Therefore, equation (9) for arbitrary values of the polytropic index $n$ can be written in the form

$$
Y(\xi, \theta)=1+\frac{\Omega^{2} \xi^{2}}{6}\left(1-P_{2}(t)\right)+\frac{1}{4 \pi} \int Y^{n}\left(\xi^{\prime}, \theta^{\prime}\right) Q\left(\boldsymbol{\xi}, \boldsymbol{\xi}^{\prime}\right) d \boldsymbol{\xi}^{\prime}
$$

\section{The Emden's equation}

The partial case $\Omega=0$ corresponds to the polytropic star with spherical symmetry and the equation (7) becomes the one-dimensional one-parametric equation

$$
\Delta_{\xi} y(\xi)=-y^{n}(\xi)
$$

Mathematical Modeling and Computing, Vol. 6, No. 2, pp. 153-172 (2019) 
with the boundary conditions $y(0)=1, d y / d \xi=0$ at $\xi=0$, as well as the physical condition $y(\xi) \geqslant 0$. The dimensionless stellar radius $\xi_{1}$ is a smaller root of the equation $y(\xi)=0$. All stellar characteristics are determined by the polytropic index $n \geqslant 0$. The value $n=5$ is critical: at $n<5$ the solutions of equation (16) are the alternating-sign functions of $\xi$, and at $n \geqslant 5$ they are positive in all range $0 \leqslant \xi \leqslant \infty$ and the boundary condition on the stellar surface is not fulfilled $\left(\xi_{1}(5)=\infty\right.$, that corresponds to a star without external border). Henceforward we only consider the models with $n \leqslant 5$ $(\nu \geqslant 6 / 5)$. After integration over kernel angular variables $Q\left(\boldsymbol{\xi}, \boldsymbol{\xi}^{\prime}\right)$ the equation $(9)$ takes the form

$$
y(\xi)=1+\int_{0}^{\xi}\left\{\frac{\left(\xi^{\prime}\right)^{2}}{\xi}-\xi^{\prime}\right\} y^{n}\left(\xi^{\prime}\right) d \xi^{\prime} .
$$

The characteristic features of solutions for equations (16), (17) are well known [12]. They are illustrated by the accurate analytical solutions at $n=0 ; 1 ; 5$, namely

$$
\begin{aligned}
& y_{0}(\xi)=1-\xi^{2} / 6 ; \quad \xi_{1}(0)=\sqrt{6} ; \quad y_{1}(\xi)=\frac{1}{\xi} \sin \xi ; \quad \xi_{1}(1)=\pi \\
& y_{5}(\xi)=\left\{1+\xi^{2} / 3\right\}^{-1 / 2} ; \quad \xi_{1}(5)=\infty
\end{aligned}
$$

For other values of index $n$ we can get the solution through numerical integration of the equation (16) or (17). In the range of small values $\xi$ the Emden's functions have such series expansion [12]:

$$
y_{n}(\xi)=1-\frac{1}{3 !} \xi^{2}+\frac{n}{5 !} \xi^{4}-\frac{n(8 n-5)}{3 \cdot 7 !} \xi^{6}+\ldots
$$

In the case $n>1$ the equation (16) can be "linearized", neglecting the term $y_{n}^{n}(\xi)$ in the surface stellar region. The solution of equation $\Delta_{\xi} y_{n}(\xi)=0$ is the function

$$
y_{n}(\xi)=\beta_{2}(n)\left(\frac{1}{\xi}-\frac{1}{\xi_{1}(n)}\right) \simeq \frac{\beta_{2}(n)}{\xi_{1}^{2}(n)}\left(\xi_{1}(n)-\xi\right),
$$

which determines the asymptotic of solution in the vicinity $\xi_{1}(n)$. Herewith

$$
\beta_{2}(n)=\int_{0}^{\xi_{1}(n)} \xi^{2} y_{n}^{n}(\xi) d \xi=\xi_{1}^{2}(n)\left|\frac{d y_{n}}{d \xi}\right|_{\xi_{1}(n)}
$$

is characteristic parameter of the polytropic model, that determines its mass and energy. The important feature of the solutions of equilibrium equation at $n \neq 0$ are the presence of the inflection point $\xi_{2}(n)$, where $d^{2} y_{n} / d \xi^{2}=0$. This point divides the range of variable $\xi$ with different value of second derivative: at $\xi<\xi_{2}(n)$ the curve $y_{n}(\xi)$ is convex and in the region $\xi>\xi_{2}(n)$ is concave. In the first region the expansion in series by powers of $\xi^{2}$ is applicable and in the second one - series

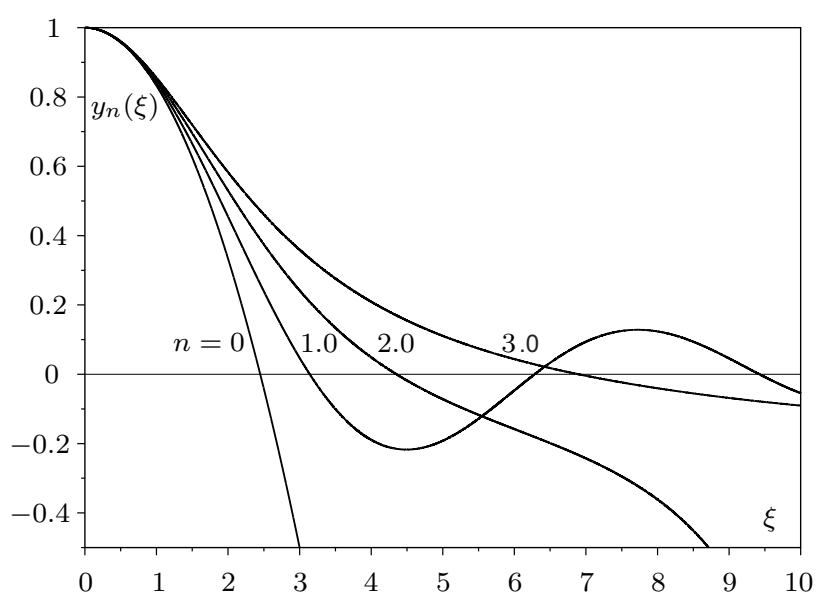

Fig. 1. The solutions of Emden's equation for the polytropic index $0 \leqslant n \leqslant 3.0$. of type

$$
y_{n}(\xi)=\sum_{m=1}^{\infty} a_{n, m}\left(\xi_{1}(n)-\xi\right)^{m} .
$$

This series are also valid in the region $\xi>\xi_{1}(n)$ and can be used when describing the stars with axial rotation. The nature of the solutions of equations (16), (17) are illustrated in Fig. 1. 
The dependencies of the values $\xi_{1}(n), \xi_{2}(n), \beta_{2}(n)$ and $\beta_{4}(n)$ on polytropic index are shown in the Table 1.

Table 1. The parameters of Emden's polytropic models.

\begin{tabular}{|c|c|c|c|c|c|c|c|c|}
\hline$n$ & 0 & 0.25 & 0.5 & 1.0 & 1.5 & 2.0 & 2.5 & 3.0 \\
\hline \hline$\xi_{1}(n)$ & 2.4495 & 2.5921 & 2.7527 & 3.1416 & 3.6538 & 4.3529 & 5.3553 & 6.8969 \\
\hline$\xi_{2}(n)$ & - & 2.4780 & 2.3470 & 2.0810 & 1.8780 & 1.7210 & 1.5970 & 1.4960 \\
\hline$\beta_{2}(n)$ & 4.8990 & 4.2579 & 3.7887 & 3.1416 & 2.7141 & 2.4111 & 2.1872 & 2.0182 \\
\hline$\beta_{4}(n)$ & 17.6371 & 15.5178 & 14.0352 & 12.1567 & 11.1197 & 10.6110 & 10.5197 & 10.8516 \\
\hline
\end{tabular}

As can be seen from the formulae (18), the function $y_{1}(\xi)$ coincides with the spherical Bessel function of first kind $j_{0}(\xi)$ [14]. The functions $y_{n}(\xi)$ at $n>1$ in the asymptotic $\xi \ll 1$ also coincide with the function $j_{0}(\xi)$. The solutions of equation (16) was shown in Fig. 1, these solutions are changeable functions $\xi$ : if $n$ is even, then the equation $y_{n}(\xi)=0$ has a single root $\xi_{1}(n)$, in the case of odd $n$ this equation has many roots $\xi_{m}(n)$ and the functions $y_{n}(\xi)$ are oscillating. In general the functions $y_{n}(\xi)$ in the region of $\xi \leqslant \xi_{1}(n)$ are analogues of the function $j_{0}(\xi)$.

According to the formulae (10) the mass, volume and moment of inertia of the polytropic star without rotation are determined by expressions:

$$
\begin{aligned}
M(n, 0) & =4 \pi \rho_{c} \lambda_{n}^{3} \int_{0}^{\xi_{1}(n)} \xi^{2} y_{n}^{n}(\xi) d \xi=4 \pi \rho_{c} \lambda_{n}^{3} \beta_{2}(n) ; \quad V(n, 0)=\frac{4 \pi}{3}\left(\lambda_{n} \xi_{1}(n)\right)^{3} \\
I(n, 0) & =\frac{8}{3} \pi \lambda_{n}^{5} \rho_{c} \int_{0}^{\xi_{1}(n)} \xi^{4} y_{n}^{n}(\xi) d \xi=\frac{8}{3} \pi \lambda_{n}^{5} \rho_{c} \beta_{4}(n) .
\end{aligned}
$$

\section{The models with axial rotation. Linear equations}

To reveal the basic differences of the solutions of equations (7) and (15) on the Emden's functions, we consider partial cases $n=0$ and $n=1$, when these equations are linear and allow the solutions in analytical form, unlike the works $[7,8]$. Note, that the case $n=0$ in these works was not considered.

\subsection{Polytrope $n=0$}

This simple model with constant density $\left(\rho(\mathbf{r})=\rho_{c}\right)$ corresponds to incompressible fluid and the solution of equilibrium equation only determines shape and size of the object. It is used in the theory of homogeneous rotational spheroids and ellipsoids [13]. We consider dimensionless angular velocity (but not eccentricity) as the independent parameter and we determine mass and shape of model surface in the region of valid values of angular velocity.

According to the formulae (14), (15) at the solution of equilibrium equation takes the form

$$
Y_{0}(\xi, \theta)=y_{0}(\xi)+\frac{\xi^{2}}{6} \Omega^{2}\left(1-P_{2}(t)\right)-\frac{\xi^{2}}{2} P_{2}(t) I(e),
$$

where $y_{0}(\xi)$ is the Emden's function with polytropic index $n=0$. From the condition $Y_{0}(\xi, \theta)=0$ we find the surface equation

$$
\xi_{0}(\theta)=\sqrt{6}\left\{1-\Omega^{2}\left(1-P_{2}(t)\right)+3 P_{2}(t) I(e)\right\}^{-1 / 2} .
$$

Having determined from that formula polar and equatorial radii and taking advantage of the ratio $1-e^{2}=\xi_{p}^{2} / \xi_{e}^{2}$ we obtain the equation

$$
e^{2}+\left(3 e^{2}-\frac{9}{2}\right) I(e)=\frac{3}{2} \Omega^{2}
$$


which defines the relation of eccentricity with angular velocity. In the approximation $e^{2} \ll 1$

$$
I(e)=\frac{2}{15} e^{2}+\frac{8}{105} e^{4}+\ldots, \quad e^{2}=\frac{15}{4} \Omega^{2}-\frac{225}{112} \Omega^{4}+\ldots, \quad I(e)=\frac{1}{2} \Omega^{2}-\frac{2}{15} \Omega^{4}+\ldots
$$

In this approximation

$$
\begin{aligned}
\xi_{0}(\theta) & =\sqrt{6}\left\{1-\Omega^{2}+\frac{5}{2} P_{2}(t)\left[\Omega^{2}-\frac{4}{25} \Omega^{4}\right]+\ldots\right\}^{-1 / 2} \\
& =\sqrt{6}\left\{1+\frac{\Omega^{2}}{2}\left[1-\frac{5}{2} P_{2}(t)\right]+\frac{3}{8} \Omega^{4}\left[1-\frac{67}{15} P_{2}(t)+\frac{25}{4} P_{2}^{2}(t)\right]+\ldots\right\},
\end{aligned}
$$

in connection with that

$$
\xi_{p} \simeq \sqrt{6}\left\{1-\frac{3}{4} \Omega^{2}+\frac{167}{160} \Omega^{4}+\ldots\right\}, \quad \xi_{e} \simeq \sqrt{6}\left\{1+\frac{9}{8} \Omega^{2}+\frac{1151}{640} \Omega^{4}+\ldots\right\} .
$$

It follows also, that mass and volume of the polytropic star with index $n=0$ are determined by the expression

$$
M(0, \Omega)=M(0,0) f(\Omega), \quad V(0, \Omega)=V(0,0) f(\Omega), \quad f(\Omega) \cong 1+\frac{3}{2} \Omega^{2}+\frac{11}{2} \Omega^{4}+\ldots,
$$

where $M(0,0)$ and $V(0,0)$ are mass and volume without rotation.

To investigate the dependence of stellar characteristics on rotation velocity in large range, we found the solution of equation (21) regarding to the eccentricity by the numerical method. It turns out that the real solutions exist only in the range $0 \leqslant \Omega \leqslant \Omega_{\max }=0.47399 \ldots$ and the eccentricity is an ambiguous function of velocity (Fig. 2). The value $\Omega_{\max }$ corresponds to the $e\left(\Omega_{\max }\right)=0.92995 \ldots$

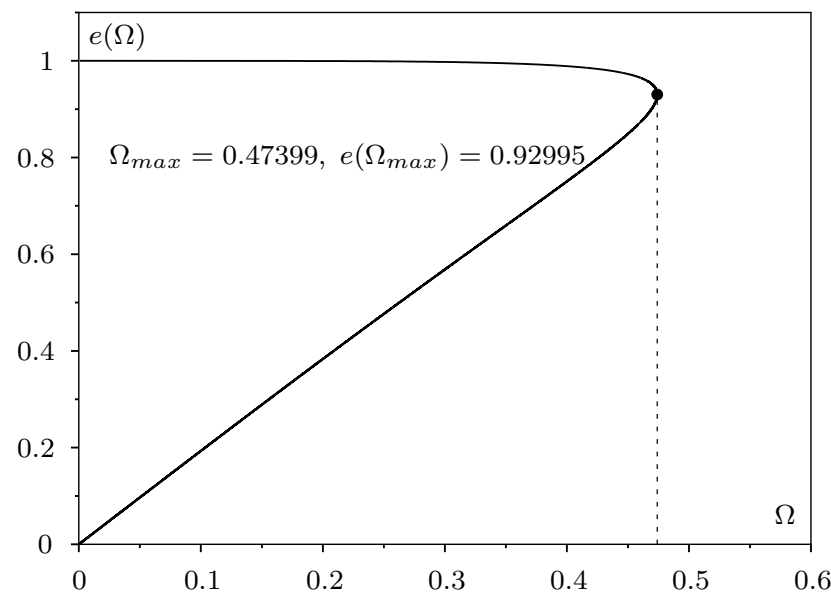

Fig. 2. Dependence of the eccentricity $e(\Omega)$ on the angular velocity $\Omega$.

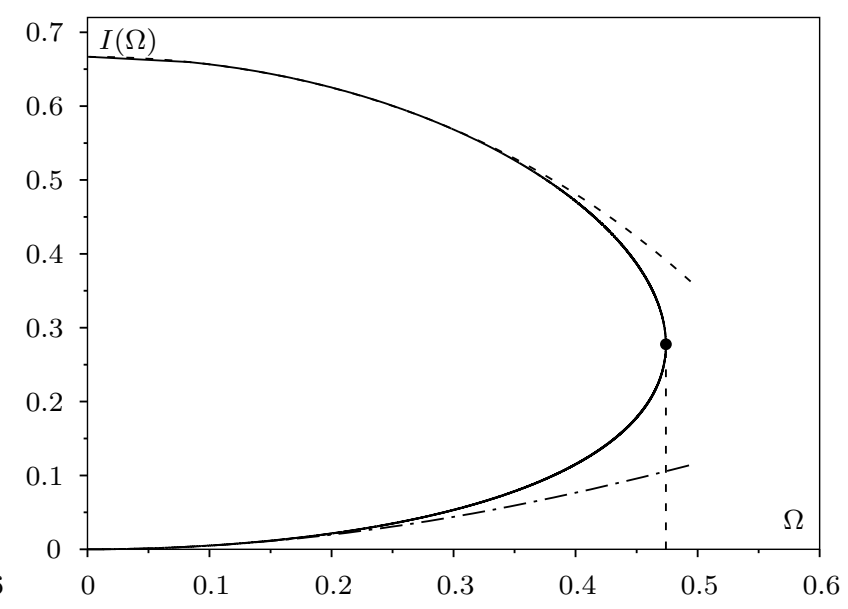

Fig. 3. Dependence of the function $I(\Omega)$ on angular velocity $\Omega$. Dashed-dot curve corresponds to small velocity (small value of the eccentricity), dashed small velocity at large values of the eccentricity.

The configurations which belong to the region $0<e \leqslant e\left(\Omega_{\max }\right)$ are the typical ellipsoidal structures, and those which belong to the range $e\left(\Omega_{\max }\right)<e \leqslant 1.0$ are similar to disk structures. This confirm the existence of a point of bifurcation in the equation (7) relative to the angular velocity (see [13]). The dependence $I(\Omega) \equiv I(e(\Omega))$ on rotational velocity is shown in Fig. 3. Herewith $I\left(e\left(\Omega_{\max }\right)\right)=0.27698$. The solid curve in Fig. 3 corresponds to dependence, which was depicted in Fig. 2, and dashed approximation of small velocities (form. (22)). 
The dimensionless equatorial and polar radii are determined from the ratio (20)

$$
\xi_{e}(\Omega)=\sqrt{6}\left\{1-\frac{3}{2}\left[\Omega^{2}+I(\Omega)\right]\right\}^{-1 / 2}, \quad \xi_{p}(\Omega)=\sqrt{6}\{1+3 I(\Omega)\}^{-1 / 2}
$$

are shown in Fig. 4. The solid curves correspond to the numerical calculations in region $0 \leqslant e \leqslant e\left(\Omega_{\max }\right)$

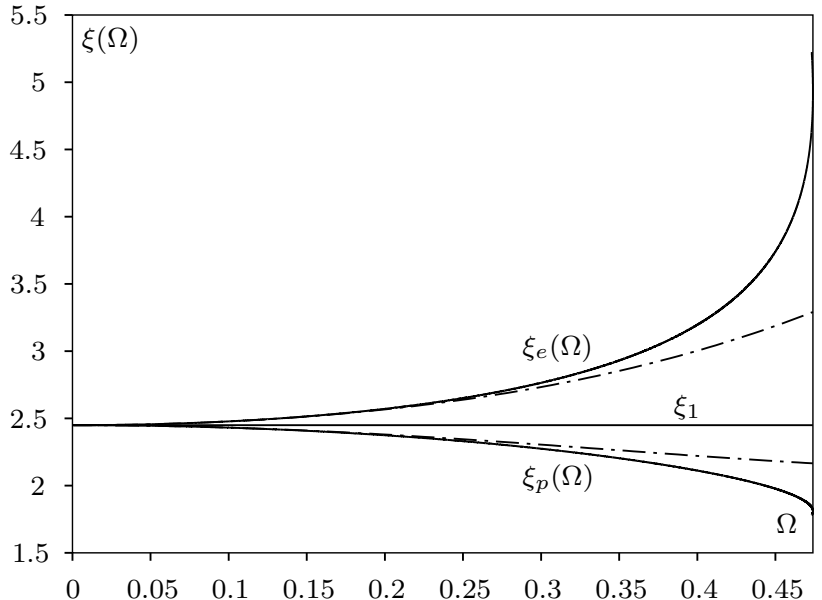

Fig. 4. Dependencies of equatorial $\xi_{e}(\Omega)$ and polar $\xi_{p}(\Omega)$ radii on angular velocity $\Omega$ (dashed-dot curves corresponds to small velocity).

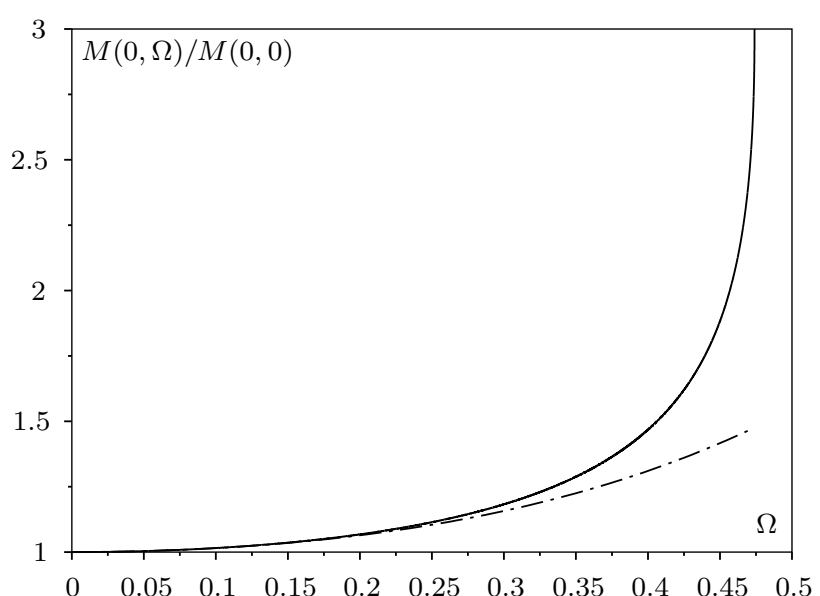

Fig. 5. Dependence of stellar polytropic mass on the rotational velocity $\Omega$ (dashed-dot curve corresponds to the formula (23)).

and dashed-dot curves - approximation of small velocities. As was shown from figure, approximation of small velocities is applicable in the range $\Omega \leqslant 0.5 \Omega_{\max }$.

Dependence of stellar mass on rotational velocity

$$
M(0, \Omega)=M(0,0) \xi_{e}^{2}(\Omega) \xi_{p}(\Omega) \xi_{1}^{-3}(0)
$$

is shown in Fig. 5. Herewith dashed-dot curve corresponds to small velocity according to the formula (23).

In the range $e\left(\Omega_{\max }\right) \leqslant e \leqslant 1$

$$
I(\Omega) \approx \frac{2}{3}-\Omega^{2}-\Omega^{4}+\ldots,
$$

which is shown with dashed curve in Fig. 3. Therefore according to the formulae (24) in region of large values of the eccentricity and small values of rotational velocity

$$
\xi_{e}(\Omega) \Rightarrow \frac{2}{\Omega^{2}}+\ldots, \quad \xi_{p}(\Omega) \Rightarrow \sqrt{2},
$$

which corresponds to the disk of constant thickness and large radius.

\subsection{Polytrope $n=1$}

In this case we use both differential and integral form of the mechanical equilibrium equation. It is convenient to rewrite the equation (7) in the form of such inhomogeneous equation

$$
\begin{aligned}
& \Delta(\xi, \theta) \varphi(\xi, \theta)+\varphi(\xi, \theta)=-\frac{\Omega^{2}}{4} \xi^{2} \sin ^{2} \theta \\
& \varphi(\xi, \theta)=Y_{1}(\xi, \theta)-\frac{\Omega^{2}}{4} \xi^{2} \sin ^{2} \theta
\end{aligned}
$$


In the corresponding homogeneous equation the variables are separated and its general solution

$$
\varphi(\xi, \theta)=\sum_{l=0}^{\infty} B_{2 l} j_{2 l}(\xi) P_{2 l}(t)
$$

where $j_{2 l}(\xi)$ is the spherical Bessel function of first kind [14], $B_{2 l}$ are the integration constants. The substitution $\varphi(\xi, \theta)=\Omega^{2} \tilde{\varphi}(\xi, \theta)$ allows to get rid of the parameter $\Omega^{2}$ in the equation (25):

$$
\Delta(\xi, \theta) \tilde{\varphi}(\xi, \theta)+\tilde{\varphi}(\xi, \theta)=-\frac{\xi^{2}}{4} \sin ^{2} \theta .
$$

We find the partial solution of this equation in the form

$$
\tilde{\varphi}(\xi, \theta)=\sum_{l=2}^{\infty} b_{2 l}[\xi \sin \theta]^{2 l}
$$

Using the equality

$$
\Delta(\xi, \theta)\{\xi \sin \theta\}^{2 l}=(2 l)^{2}\{\xi \sin \theta\}^{2 l-2},
$$

we find, that

$$
b_{2 l}=(-1)^{l-1} 2^{-2 l}(l !)^{-2},
$$

therefore

$$
\frac{1}{4} \xi^{2} \sin ^{2} \theta+\tilde{\varphi}(\xi, \theta)=1-J_{0}(\xi \sin \theta)
$$

where $J_{0}(z)$ is the Bessel function of the zero kind [14]. From the differential equation for the function $Y_{1}(\xi, \theta)$ it follows the asymptotic behavior

$$
Y_{1}(\xi, \theta) \Rightarrow 1-\frac{\xi^{2}}{6}+\frac{\Omega^{2} \xi^{2}}{4} \sin ^{2} \theta+\ldots
$$

at $\xi \rightarrow 0$, therefore the general solution is such:

$$
Y_{1}(\xi, \theta)=y_{1}(\xi)+\Omega^{2}\left\{1-J_{0}(\xi \sin \theta)\right\}+\sum_{l=1}^{\infty} B_{2 l} j_{2 l}(\xi) P_{2 l}(t),
$$

where $y_{1}(\xi)=j_{0}(\xi)$ is the Emden's function for the polytrope with index $n=1$.

It is easy to see that the function $J_{0}(\xi \sin \theta)$ has the same expansion for the Legendre polynomials $(t=\cos \theta)$ and spherical Bessel functions:

$$
\begin{aligned}
& J_{0}(\xi \sin \theta)=\sum_{l=0}^{\infty} D_{2 l} j_{2 l}(\xi) P_{2 l}(t) ; \\
& D_{0}=1 ; \quad D_{2}=\frac{5}{2} ; \quad D_{4}=\frac{27}{8} ; \quad \ldots
\end{aligned}
$$

In this regard the solution can be represented in equivalent form, namely

$$
Y_{1}(\xi, \theta)=y_{1}(\xi)+\Omega^{2}\left(1-y_{1}(\xi)\right)+\sum_{l=1}^{\infty} A_{2 l} j_{2 l}(\xi) P_{2 l}(t),
$$

where new constants $A_{2 l}=B_{2 l}-\Omega^{2} D_{2 l}$ is introduced. From the other side, according to the equation $(15)$

$$
Y_{1}(\xi, \theta)=1+\frac{\Omega^{2} \xi^{2}}{6}\left(1-P_{2}(t)\right)+\frac{1}{4 \pi} \int Y_{1}\left(\xi^{\prime}, \theta^{\prime}\right) Q\left(\boldsymbol{\xi}, \boldsymbol{\xi}^{\prime}\right) d \boldsymbol{\xi}^{\prime}
$$


The equation (27) is non-homogeneous, therefore it can be used to find the integration constants $A_{2 l}$. Substituting in it the expression (26), we get the expression

$$
\sum_{l=1}^{\infty} A_{2 l} j_{2 l}(\xi) P_{2 l}(t)=-P_{2}(t) \frac{\Omega^{2}}{6} \xi^{2}\{1+3 I(e)\}+\frac{1}{4 \pi} \sum_{l=1}^{\infty} A_{2 l} \int j_{2 l}\left(\xi^{\prime}\right) P_{2 l}\left(t^{\prime}\right) Q\left(\boldsymbol{\xi}, \boldsymbol{\xi}^{\prime}\right) d \boldsymbol{\xi}^{\prime}
$$

We will perform integration over variables $\xi^{\prime}, t^{\prime}, \varphi^{\prime}$ in the form of the rotational ellipsoid model with the eccentricity $e$ and the equatorial radius $\xi_{e}$, as in the case $n=0$, expanding the kernel $Q\left(\boldsymbol{\xi}, \boldsymbol{\xi}^{\prime}\right)$ in series of Legendre polynomials:

$$
\begin{aligned}
\frac{1}{4 \pi} \sum_{l=1}^{\infty} A_{2 l} \int j_{2 l}\left(\xi^{\prime}\right) & P_{2}\left(t^{\prime}\right) Q\left(\boldsymbol{\xi}, \boldsymbol{\xi}^{\prime}\right) d \boldsymbol{\xi}^{\prime}=\sum_{l=1}^{\infty} \frac{A_{2 l}}{4 l+1} \frac{P_{2 l}(t)}{\xi^{1+2 l}} \int_{0}^{\xi}\left(\xi^{\prime}\right)^{2+2 l} j_{2 l}\left(\xi^{\prime}\right) d \xi^{\prime} \\
+ & \frac{1}{2} \sum_{l=1}^{\infty} A_{2 l} P_{2 l}(t) \xi^{2 l} \int_{-1}^{+1} P_{2 l}^{2}\left(t^{\prime}\right) d t^{\prime} \int_{\xi}^{\xi_{0}\left(t^{\prime}\right)} j_{2 l}\left(\xi^{\prime}\right)\left(\xi^{\prime}\right)^{1-2 l} d \xi^{\prime} \\
+ & \frac{1}{2} \sum_{l, m=1}^{\infty} A_{2 l} P_{2 m}(t) \xi^{2 m}\left(1-\delta_{m, l}\right) \int_{-1}^{+1} P_{2 l}\left(t^{\prime}\right) P_{2 m}\left(t^{\prime}\right) d t^{\prime} \int_{\xi}^{\xi_{0}\left(t^{\prime}\right)} j_{2 l}\left(\xi^{\prime}\right)\left(\xi^{\prime}\right)^{1-2 m} d \xi^{\prime}
\end{aligned}
$$

where $\delta_{n, l}$ is the Kronecker symbol and $\xi_{0}\left(t^{\prime}\right)$ is determined by formula (13).

Integration over the variable $\xi^{\prime}$ is performed in analytical form using the equation for the function $j_{2 l}(\xi)$, as well as recurrence formulae

$$
\begin{aligned}
& (4 l+1) j_{2 l}(\xi)=\xi\left\{j_{2 l+1}(\xi)+j_{2 l-1}(\xi)\right\} \\
& \frac{d}{d \xi} j_{l}(\xi)=j_{l-1}(\xi)-\frac{l+1}{\xi} j_{l}(\xi) ; \quad l \geqslant 1 .
\end{aligned}
$$

Equating the coefficients at the same factors $\xi^{2 l} P_{2 l}(t)$ in left and right sides of the expression (28), we obtain the system of linear equations for the constants $A_{2 l}$ :

$$
\begin{aligned}
& A_{2} S_{2,2}+A_{4} S_{2,4}+A_{6} S_{2,6}+\ldots=-\frac{\Omega^{2}}{6}(1+3 I(e)) \\
& A_{2} B_{4,2}+A_{4} S_{4,4}+A_{6} B_{4,6}+\ldots=0 ; \\
& A_{2} C_{6,2}+A_{4} C_{6,4}+A_{6} S_{6,6}+\ldots=0 ; \quad \ldots
\end{aligned}
$$

The coefficients $S_{2 l, 2 l}, S_{2 l, 2 n}, B_{2 n, 2 l}, C_{2 n, 2 l}, \ldots$ are functions of the parameters $e, \xi_{e}$ and determined by such expressions:

$$
\begin{gathered}
S_{2 l, 2 l}=\int_{0}^{1} P_{2 l}^{2}(t) \xi_{0}^{1-2 l} j_{2 l-1}\left(\xi_{0}\right) d t ; \quad S_{2,4}=\int_{0}^{1} P_{2}(t) P_{4}(t) \xi_{0}^{-1}\left\{j_{3}\left(\xi_{0}\right)+2 \xi_{0}^{-1} j_{2}\left(\xi_{0}\right)\right\} d t \\
S_{2,6}=\int_{0}^{1} P_{2}(t) P_{6}(t) \xi_{0}^{-1}\left\{j_{5}\left(\xi_{0}\right)+4 \xi_{0}^{-1} j_{4}\left(\xi_{0}\right)+8 \xi_{0}^{-2} j_{3}\left(\xi_{0}\right)\right\} d t \\
B_{4,2}=-\int_{0}^{1} P_{4}(t) P_{2}(t)\left\{\int_{\xi_{1}(n)}^{\xi_{0}} j_{2}\left(\xi^{\prime}\right)\left(\xi^{\prime}\right)^{-3} d \xi^{\prime}\right\} d t ; B_{4,6}=\int_{0}^{1} P_{4}(t) P_{6}(t) \xi_{0}^{-3}\left\{j_{5}\left(\xi_{0}\right)+2 \xi_{0}^{-1} j_{4}\left(\xi_{0}\right)\right\} d t \\
C_{6,2}=-\int_{0}^{1} P_{6}(t) P_{2}(t)\left\{\int_{\xi_{1}(n)}^{\xi_{0}} j_{2}\left(\xi^{\prime}\right)\left(\xi^{\prime}\right)^{-5} d \xi^{\prime}\right\} d t \\
C_{6,4}=-\int_{0}^{1} P_{6}(t) P_{4}(t)\left\{\int_{\xi_{1}(n)}^{\xi_{0}} j_{4}\left(\xi^{\prime}\right)\left(\xi^{\prime}\right)^{-5} d \xi^{\prime}\right\} d t \ldots
\end{gathered}
$$


As was shown from the expressions (30),

$$
S_{2 l, 2 l} \approx \tilde{S}_{2 l, 2 l}=(4 l+1)^{-1} \xi_{1}^{-1} j_{2 l-1}\left(\xi_{1}\right),
$$

where $\xi_{1}=\pi$ is the dimensionless radius in the Emden's approximation. Non-diagonal coefficients (30) are the small values which are proportional to the degrees of squared eccentricity (to $\Omega^{2}$ ). The approximation of E. Milne-S. Chandrasekhar $[7,8]$ corresponds to

$$
\tilde{A}_{2}=-\frac{\Omega^{2}}{6}\left\{\tilde{S}_{2,2}\right\}^{-1}, \quad \tilde{A}_{4}=\tilde{A}_{6}=\ldots=0
$$

that mean full neglect of stellar surface variation because of of rotation while determining of constants $A_{2 l}$.

The coefficients $A_{2 l}$ are the functions of angular velocity, as well as the eccentricity $e$ and the equatorial radius $\xi_{e}$, which are the same functions of angular velocity. Therefore the problem arises of self-consistent determining of geometrical parameters of rotational ellipsoid. From the condition

$$
\Omega^{2}+\left(1-\Omega^{2}\right) y_{1}\left(\xi_{0}(t)\right)+\sum_{l=1}^{\infty} A_{2 l} j_{2 l}\left(\xi_{0}(t)\right) P_{2 l}(t)=0
$$

we will find the equation of stellar surface at a fixed value of the angular velocity $\xi_{0}(t) \equiv \xi_{0}(t \mid \Omega)$. The root of equation at $t=1$ determines the polar radius $\xi_{p}(\Omega) \equiv \xi_{0}(1 \mid \Omega)$ and the root at $t=0$ takes the equatorial radius $\xi_{e}(\Omega) \equiv \xi_{0}(0 \mid \Omega)$ at $0 \leqslant \Omega \leqslant \Omega_{\max }$. The equation

$$
e^{2}(\Omega)=1-\left[\frac{\xi_{0}(1 \mid \Omega)}{\xi_{0}(0 \mid \Omega)}\right]^{2}
$$

determines the dependence of eccentricity $e(\Omega)$ on angular velocity. The system of equations (29), (32), (33), in which $\Omega$ is an independent parameter, determines the dependence $e(\Omega), \xi_{e}(\Omega), \xi_{p}(\Omega)$ and $A_{2 l}(\Omega)(1 \leqslant l \leqslant 3)$ on angular velocity. The system solves numerically by the method of successive approximations. The algorithm of successive iterations are as follows. At the initial value $\Omega_{1} \ll 1$ in zero approximation values of $\xi_{e}(\Omega)=\xi_{p}(\Omega)$ we determine from the equation (32) at $A_{2 l}=0$. Next we find the values $S_{2 l, 2 l}, S_{2,4}, \ldots, C_{6,4}$ and solve the system of equations (29). In the next iteration we find $\xi_{p}(\Omega)$ and $\xi_{e}(\Omega)$ from the equation (32) with help of found coefficients $A_{2 l}$ and calculate the eccentricity $e(\Omega)$. We calculate again $S_{2 l, 2 l}, \ldots, C_{6,4}$ and etc. The final values $A_{2 l}\left(\Omega_{1}\right)$ are used as the zero successive approximation for the calculation of the characteristics at $\Omega_{2}=\Omega_{1}+\Delta \Omega$ and etc. Obtained in this way integration constants and ellipsoid's characteristics are shown in Table 2 .

The maximal value of angular velocity determines from condition of the disappearance of solution of the equation (32) at $t=0$ : at the value $\Omega_{\max }$ the equatorial radius becomes infinite and the polar remains finite. This is an instability point, at which the leakage of the material occurs from the vicinity of the equator according to the condition (8). In the approximation $A_{2 l}=0$ at $l \geqslant 2$ we find that $\Omega_{\max }=0.29770 \ldots$ Considering $A_{2}(\Omega), A_{4}(\Omega), A_{6}(\Omega)$ we get the value $\Omega_{\max }=0.24607 \ldots$

Dependence of values $e(\Omega), \xi_{e}(\Omega), \xi_{p}(\Omega), A_{2}(\Omega)-A_{6}(\Omega)$, as well as the factor

$$
\eta(1, \Omega)=M(1, \Omega) / M(1,0)
$$

on the angular velocity is shown in the Tab. 2. Here $M(1, \Omega)$ denotes the mass of the rotational ellipsoid in the approximation $A_{2 l}=0$ at $l \geqslant 4$ and $M(1,0)$ is the mass of polytropic star without rotation. Also the dependence on angular velocity of the dimensionless moment of inertia is

$$
\zeta(1, \Omega)=I(1, \Omega) / I(1,0)
$$

where $I(1,0)=8 \pi^{2} \lambda^{5} \rho_{c}\left(\pi^{2}-6\right) / 3$ is the moment of inertia in the Emden's model. 
Table 2. Dependence of the model characteristics with index $n=1$ on angular velocity.

\begin{tabular}{|c|c|c|c|c|c|c|c|c|}
\hline$\Omega$ & $e(\Omega)$ & $\xi_{p}(\Omega)$ & $\xi_{e}(\Omega)$ & $A_{2}(\Omega)$ & $A_{4}(\Omega)$ & $A_{6}(\Omega)$ & $\eta(n, \Omega)$ & $\zeta(n, \Omega)$ \\
\hline$\overline{0.01000}$ & 0.02739 & 3.14112 & 3.14230 & -0.00082 & $6.10775 \cdot 10^{-7}$ & $-8.02713 \cdot 10^{-10}$ & 1.00023 & 1.00062 \\
\hline 0.02000 & .05478 & 3.13971 & 3.14443 & -0.00329 & $9.796 \cdot 10^{-6}$ & $-5.15628 \cdot 10^{-8}$ & 1.00092 & 1.00249 \\
\hline 0.03000 & 0.08219 & 3.13734 & 3.14799 & -0.00743 & $4.97925 \cdot 10^{-5}$ & $-5.91249 \cdot 10^{-7}$ & 1.00207 & 1.00563 \\
\hline 0.04000 & 0.10961 & 3.13402 & 3.15302 & -0.01324 & 0.000158264 & $-3.3532 \cdot 10^{-6}$ & 1.00369 & 1.01006 \\
\hline 0.05000 & 0.13706 & 3.12973 & 3.15955 & -0.02076 & 0.000389238 & $-1.2947 \cdot 10^{-5}$ & 1.00580 & 1.01583 \\
\hline 0.06000 & 0.16455 & 3.12447 & 3.16765 & -0.03003 & 0.000814471 & $-3.92393 \cdot 10^{-5}$ & 1.00839 & 1.02298 \\
\hline 0.07000 & 0.19208 & 3.11820 & 3.17737 & -0.04109 & 0.00152534 & -0.00010072 & 1.01150 & 1.03158 \\
\hline 0.08000 & 0.21967 & 3.11092 & 3.18880 & -0.05399 & 0.00263535 & -0.000229121 & 1.01513 & 1.04172 \\
\hline 0.09000 & 0.24733 & 3.10259 & 3.20205 & -0.06881 & 0.00428336 & -0.000475679 & 1.01933 & 1.05351 \\
\hline 0.10000 & 0.27507 & 3.09318 & 3.21725 & -0.08564 & 0.00663789 & -0.000919578 & 1.02410 & 1.06707 \\
\hline 0.11000 & 0.30291 & 3.08266 & 3.23456 & -0.10456 & 0.00990262 & -0.00167936 & 1.02951 & 1.08256 \\
\hline 0.12000 & 0.33087 & 3.07097 & 3.25416 & -0.12569 & 0.0143237 & -0.00292837 & 1.03557 & 1.10016 \\
\hline 0.13000 & 0.35900 & 3.05807 & 3.27632 & -0.14919 & 0.0201994 & -0.00491599 & 1.04237 & 1.12011 \\
\hline 0.14000 & 0.38731 & 3.04388 & 3.30131 & -0.17521 & 0.0278935 & -0.00799698 & 1.04994 & 1.14270 \\
\hline 0.15000 & 0.41586 & 3.02832 & 3.32953 & -0.20397 & 0.0378538 & -0.0126729 & 1.05839 & 1.16830 \\
\hline 0.16000 & 0.44471 & 3.01127 & 3.36147 & -0.23574 & 0.0506373 & -0.0196514 & 1.06782 & 1.19736 \\
\hline 0.17000 & 0.47394 & 2.99259 & 3.39779 & -0.27082 & 0.0669518 & -0.0299343 & 1.07834 & 1.23047 \\
\hline 0.18000 & 0.50367 & 2.97208 & 3.43938 & -0.30965 & 0.0877136 & -0.0449518 & 1.09014 & 1.26843 \\
\hline 0.19000 & 0.53407 & 2.94946 & 3.48752 & -0.35278 & 0.11415 & -0.0667796 & 1.10343 & 1.31232 \\
\hline 0.20000 & 0.56538 & 2.92430 & 3.54414 & -0.40103 & 0.147978 & -0.0985096 & 1.11855 & 1.36371 \\
\hline 0.21000 & 0.59802 & 2.89594 & 3.61237 & -0.45558 & 0.191751 & -0.14496 & 1.13597 & 1.42496 \\
\hline 0.22000 & 0.63273 & 2.86321 & 3.69793 & -0.51842 & 0.249659 & -0.21424 & 1.15648 & 1.50007 \\
\hline 0.23000 & 0.67114 & 2.82368 & 3.81334 & -0.59350 & 0.329832 & -0.322202 & 1.18158 & 1.59696 \\
\hline 0.24000 & 0.71852 & 2.77019 & 4.00008 & -0.69231 & 0.455201 & -0.513924 & 1.21544 & 1.73805 \\
\hline 0.24100 & 0.72446 & 2.76320 & 4.02826 & -0.70491 & 0.472987 & -0.543279 & 1.21980 & 1.75727 \\
\hline 0.24200 & 0.73086 & 2.75562 & 4.06018 & -0.71850 & 0.492654 & -0.576426 & 1.22449 & 1.77830 \\
\hline 0.24300 & 0.73793 & 2.74724 & 4.09737 & -0.73341 & 0.514881 & -0.614842 & 1.22964 & 1.80179 \\
\hline 0.24400 & 0.74604 & 2.73767 & 4.14281 & -0.75034 & 0.54095 & -0.661368 & 1.23546 & 1.82894 \\
\hline 0.24500 & 0.75612 & 2.72593 & 4.20403 & -0.77099 & 0.574031 & -0.723247 & 1.24249 & 1.86270 \\
\hline 0.24600 & 0.77450 & 2.70593 & 4.33124 & -0.80636 & 0.634619 & -0.849421 & 1.25413 & 1.92196 \\
\hline 0.24601 & 0.77507 & 2.70536 & 4.33555 & -0.80739 & 0.63648 & -0.85372 & 1.25445 & 1.92369 \\
\hline 0.24602 & 0.77563 & 2.70481 & 4.33977 & -0.80839 & 0.638275 & -0.857875 & 1.25477 & 1.92537 \\
\hline 0.24603 & 0.77626 & 2.70418 & 4.34461 & -0.80951 & 0.640319 & -0.862636 & 1.25512 & 1.92728 \\
\hline 0.24604 & 0.77702 & 2.70344 & 4.35043 & -0.81086 & 0.64275 & -0.86836 & 1.25554 & 1.92955 \\
\hline 0.24605 & 0.77800 & 2.70249 & 4.35808 & -0.81259 & 0.645908 & -0.875871 & 1.25608 & 1.93248 \\
\hline 0.24606 & 0.77959 & 2.70100 & 4.37053 & -0.81535 & 0.650937 & -0.888039 & 1.25693 & 1.93714 \\
\hline 0.24607 & 0.78685 & 2.69478 & 4.42985 & -0.82731 & 0.672619 & -0.94297 & 1.26065 & 1.95773 \\
\hline
\end{tabular}

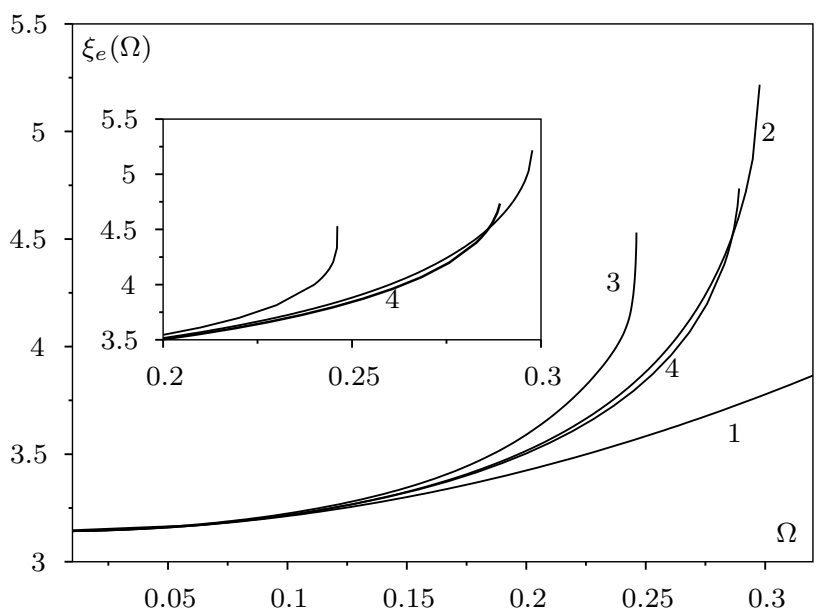

Fig. 6. Dependence of the equatorial radius $\xi_{e}(\Omega)$ on rotation velocity $\Omega$ in different approximations. Curve 1 built on the results of works [7,8], curves 2 and 3 correspond to the formula (26): curve 2 corresponds to the approximation $A_{2 l}=0(l \geqslant 2)$, curve 3 - corresponds to inclusion the terms $A_{6}$. Curve 4 built on the results of work [9].

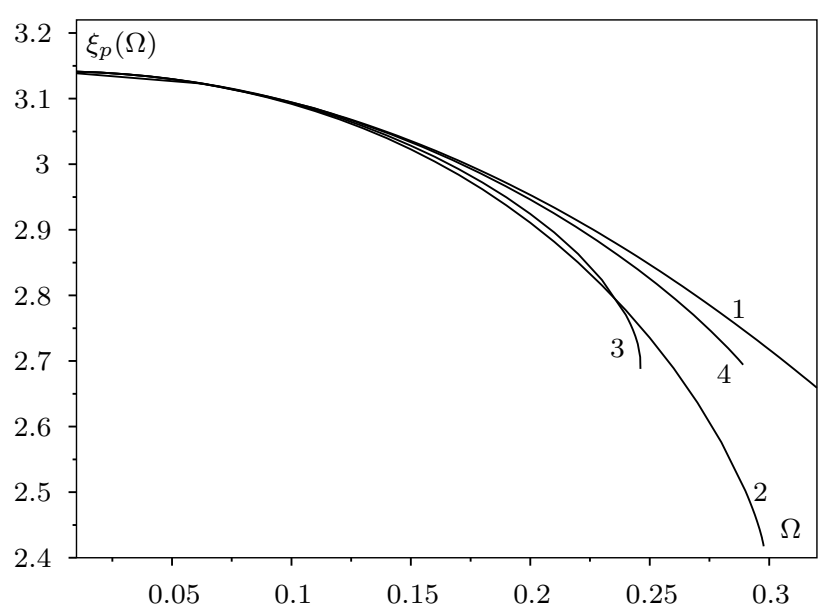

Fig. 7. Dependence of the polar radius $\xi_{p}(\Omega)$ on rotation velocity $\Omega$ in different approximations. Curve 1 built on the results of works [7,8], curves 2 and 3 correspond to the formula (26): curve 2 corresponds to the approximation $A_{2 l}=0(l \geqslant 2)$, curve 3 - corresponds to inclusion the terms $A_{6}$. Curve 4 built on the results of work [9]. 
The dependence of equatorial radius on the axial velocity in different approximations is illustrated in Fig. 6. Similarly, the dependence of polar radius on the axial velocity in the same approximations is given in Fig. 7 .

Moreover, the cross section of polytropic star according to the formula (32), as well as the ellipsoid surface according to the formula (13) at $\Omega=0.2$ are given in Fig. 8 with help of $e(\Omega)$ and $\xi_{e}(\Omega)$ from Table 2 . As we can see, there exists a slight deviation of stellar form from the exact rotational ellipsoid.

As was shown from Table 2, integration constants $A_{2}(\Omega), A_{4}(\Omega), A_{6}(\Omega), \ldots$ make alternating-sign series, moreover $\left|A_{2 l}(\Omega)\right| \sim \Omega^{2 l}$. The relative contributions of expansion terms (26) are illustrated in Figs. 6-8. The series (26) has a good convergence and sufficient number of terms of series provides more accurate values of the characteristics. In this sense the precision of numerical calculations in the work [9] is approximately of the same order that gives term $A_{2}(\Omega)$ with not very accurate calculation of constants of integration. In general, it is more important the precision of calculation of $A_{2}(\Omega)$, than taking into account terms with $A_{4}(\Omega), A_{6}(\Omega)$.

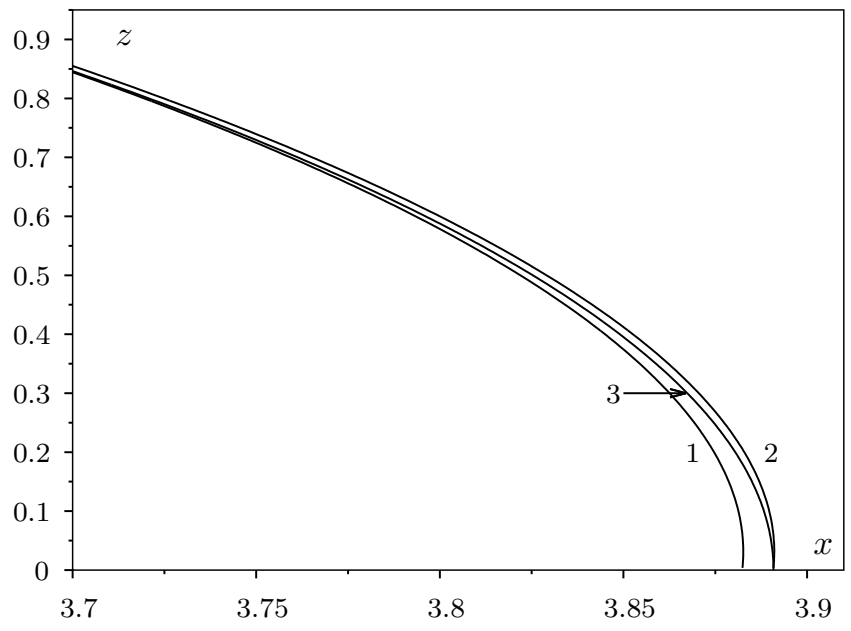

Fig. 8. Fragment of rotational ellipsoid section in the vicinity of the equator at $\Omega=0.2$. Curve 1 depicts the section of the ellipsoid surface in the approximation $A_{2 l}(l \geqslant 2)$, curve 2 corresponds to the approximation $A_{2 l}(l \geqslant 4)$, curve 3 - part of the ellipse, constructed according to the formula $(13)$, in which $\xi_{e}(\Omega)$ and $e(\Omega)$ are used from Table 2.

\section{Nonlinear equilibrium equations}

From the formulae (19) and (26) it follows the way of approximate solution of equilibrium equations at $n>1$, namely linearization of these equations, that in the case of small angular velocities was proposed in the work [7]. As was shown from the section 4 at the transition of polytrope with index $n=0$ to the polytrope with $n=1$ the maximal value of angular velocity decreases approximately in 1.9 times. From the calculation of polar and equatorial radii in the work [9] we can conclude that the approximation expression $\Omega_{\max }(n) \approx 2^{1-n} \Omega_{\max }(1)$ is fulfilled, where $\Omega_{\max }(n)$ is the maximal value of angular velocity for the polytrope with index $n$. That is a reason for the linearization of the equation (7) limiting the terms linear at $\Omega^{2}$ and using the substitution

$$
Y_{n}(\xi, \theta)=y_{n}(\xi)+\Omega^{2} \Psi_{n}(\xi, \theta) .
$$

Here $y_{n}(\xi)$ is the Emden's function for the polytrope with index $n$ and $\Psi_{n}(\xi, \theta)$ is the unknown function which satisfies the linear equation

$$
\Delta(\xi, \theta) \Psi_{n}(\xi, \theta)=1-n \cdot y_{n}^{n-1}(\xi) \Psi_{n}(\xi, \theta) .
$$

For the function $\Psi_{n}(\xi, \theta)$ it is used the substitution, which follows from the character of the solution (26):

$$
\Psi_{n}(\xi, \theta)=\psi_{n, 0}(\xi)+\sum_{l=1}^{\infty} \alpha_{2 l} \psi_{n, 2 l}(\xi) P_{2 l}(t),
$$

where $\alpha_{2 l}$ are the integration constants. Approximation of work [8] corresponds to the condition $\alpha_{2 l}=0$ at $l \geqslant 2$. Substituting the expression (36) in the equation (35), we get the independent linear 
equations for the variable $\xi$ :

$$
\begin{aligned}
& \Delta(\xi) \psi_{n, 0}(\xi)=1-n \cdot y_{n}^{n-1}(\xi) \psi_{n, 0}(\xi), \\
& \Delta(\xi) \psi_{n, 2 l}(\xi)=\left\{\frac{2 l(2 l+1)}{\xi^{2}}-n y_{n}^{n-1}(\xi)\right\} \psi_{n, 2 l}(\xi) ; \quad l \geqslant 1 .
\end{aligned}
$$

According to the asymptotic of Emden's function $y_{n}(\xi)$, expansion in series (34) and the substitution (36), the functions $\psi_{n, 0}(\xi)$ and $\psi_{n, 2 l}(\xi)$ satisfy zero limit conditions: $\psi_{n, 0}(0)=\psi_{n, 2 l}(0)=0$, $\partial \psi_{n, 0}(\xi) / \partial \xi=\partial \psi_{n, 2 l}(\xi) / \partial \xi=0$ at $\xi \rightarrow 0$. The solutions of equations for the functions $\psi_{n, 0}(\xi)$ and $\psi_{n, 2}(\xi)$ are found in the work [8] numerically in the region $0 \leqslant \xi \leqslant \xi_{1}(n)$ and are shown in Tables. This simple approach is enough for that to describe variation of surface under influence of rotation with very small angular velocity. However, for the self-consistent description of the surface of the star in all region $0 \leqslant \Omega \leqslant \Omega_{\max }$ it is necessary to find the solutions of equation (37) in the region $\xi \leqslant \xi_{e}(\Omega)$. Taking into account that $y_{n}(\xi) \rightarrow 1$ at $\xi \ll 1$ we see that $\psi_{n, 2 l}(\xi)$ is an analogue of the Bessel function $j_{2 l}(\xi)$. Passing from the variable $\xi$ to the variable $\sqrt{n} \xi$, we find that

$$
\psi_{n, 2 l}(\xi)=j_{2 l}(\sqrt{n} \xi)
$$

in the asymptotic $\xi \ll 1$. In the region of large values $\xi$, namely at $\xi>\xi_{1}(n)$ we find the other asymptotic,

$$
\psi_{n, 0}(\xi) \approx \xi^{2} / 6 ; \quad \psi_{n, 2 l}(\xi) \approx \xi^{2 l} \text { const. }
$$

The listed features facilitate the finding of the solutions of equation (37) numerically at arbitrary integer values of the polytrope index. At fractional values of the index $n$ described approach is inapplicable, otherwise $y_{n}(\xi)$ in the region $\xi>\xi_{1}(n)$ becomes negative, which follows in imaginary $y_{n}^{n-1}(\xi)$. In the case of fractional values of index $n$ the Emden's function $y_{n}(\xi)$ are not the best approximation for the solutions of equation (7) at the self-consistent description of axial rotation, which requires more precise consideration. In this paper we calculate the variation of polytrope characteristics due to axial rotation for integer values of polytrope index in the region $0 \leqslant \Omega \leqslant \Omega_{\max }(n)$.

Let's turn to the definition of integration constants $\alpha_{2 l}$, using the integral form of mechanical equilibrium equation. In $\Omega^{2}$-approximation the analogue of equations (26), (27) take such form:

$$
\begin{aligned}
y_{n}(\xi)+\Omega^{2} \psi_{2,0}(\xi)+\Omega^{2} \sum_{l=1}^{\infty} \alpha_{2 l} \psi_{n, 2 l}(\xi) P_{2 l}(t)=1+\frac{\Omega^{2} \xi^{2}}{6}\left(1-P_{2}(t)\right)+\frac{1}{4 \pi} \int d \boldsymbol{\xi}^{\prime} Q\left(\boldsymbol{\xi}, \boldsymbol{\xi}^{\prime}\right) \\
\times\left\{y_{n}^{n}\left(\xi^{\prime}\right)+n y_{n}^{n-1}\left(\xi^{\prime}\right) \Omega^{2}\left[\psi_{2,0}\left(\xi^{\prime}\right)+\sum_{l=1}^{\infty} \alpha_{2 l} \psi_{n, 2 l}\left(\xi^{\prime}\right) P_{2 l}\left(t^{\prime}\right)\right]\right\} .
\end{aligned}
$$

This equation can be simplified taking into account the equation (17) for the Emden's function $y_{n}(\xi)$. Let's also take into account that differential equation for the function $\psi_{2,0}(\xi)$ corresponds to the integral equation

$$
\psi_{2,0}(\xi)=-\frac{1}{4 \pi} \int d \boldsymbol{\xi}^{\prime} Q\left(\boldsymbol{\xi}, \boldsymbol{\xi}^{\prime}\right)\left\{1-n y_{n}^{n-1}\left(\xi^{\prime}\right) \psi_{2,0}\left(\xi^{\prime}\right)\right\} .
$$

It follows that

$$
\frac{1}{4 \pi} \int d \boldsymbol{\xi}^{\prime} Q\left(\boldsymbol{\xi}, \boldsymbol{\xi}^{\prime}\right) n y_{n}^{n-1}\left(\xi^{\prime}\right) \psi_{2,0}\left(\xi^{\prime}\right)=\psi_{2,0}\left(\xi^{\prime}\right)-\left\{\Phi_{0}(\xi, \theta)-\Phi_{0}(0,0)\right\}
$$

(see f. (14)). As a result $y_{n}(\xi)$ and $\psi_{2,0}(\xi)$ are excluded from the equation (38) and an analogue of the equation (28) is obtained:

$$
\sum_{l=1}^{\infty} \alpha_{2 l} P_{2 l}(t) \psi_{n, 2 l}(\xi)=-\frac{\xi^{2}}{6}[1+3 I(e)] P_{2}(t)+\frac{1}{4 \pi} \int Q\left(\boldsymbol{\xi}, \boldsymbol{\xi}^{\prime}\right) \sum_{l=1}^{\infty} \alpha_{2 l} P_{2 l}\left(t^{\prime}\right) n y_{n}^{n-1}\left(\xi^{\prime}\right) \psi_{n, 2 l}\left(\xi^{\prime}\right) d \boldsymbol{\xi}^{\prime}
$$

Mathematical Modeling and Computing, Vol. 6, No. 2, pp. 153-172 (2019) 
Expanding in series the kernel $Q\left(\boldsymbol{\xi}, \boldsymbol{\xi}^{\prime}\right)$ to the Legendre polynomials and integrating by variable $\boldsymbol{\xi}^{\prime}$ and equating coefficients by the same factors $\xi^{2 l} P_{2 l}(t)$ in the left and right sides of the equation, we get the system of linear equations for the integration constants:

$$
\begin{aligned}
& \alpha_{2} S_{2,2}+\alpha_{4} S_{2,4}+\alpha_{6} S_{2,6}+\ldots=-\frac{1}{6}[1+3 I(e)] ; \\
& \alpha_{2} B_{4,2}+\alpha_{4} S_{4,4}+\alpha_{6} S_{4,6}+\ldots=0 \\
& \alpha_{2} C_{6,2}+\alpha_{4} C_{6,4}+\alpha_{6} S_{6,6}+\ldots=0 .
\end{aligned}
$$

The coefficients of this system are defined by the following expressions:

$$
\begin{aligned}
& S_{2 l, 2 l}=\int_{0}^{1} P_{2 l}^{2}(t) d t \xi_{0}^{-2 l}(t)\left\{(2 l+1) \psi_{n, 2 l}\left(\xi_{0}(t)\right)+\xi_{0}(t) \frac{d}{d \xi_{0}} \psi_{n, 2 l}\left(\xi_{0}(t)\right)\right\} \\
& S_{2,2 l}=-\int_{0}^{1} P_{2}(t) P_{2 l}(t) d t \int_{\xi_{1}(n)}^{\xi_{0}(t)} n y_{n}^{n-1}\left(\xi^{\prime}\right) \psi_{n, 2 l}\left(\xi^{\prime}\right)\left(\xi^{\prime}\right)^{-1} d \xi^{\prime} ; \quad l=2 ; 3 ; \\
& B_{4,2 l}=-\int_{0}^{1} P_{4}(t) P_{2 l}(t) d t \int_{\xi_{1}(n)}^{\xi_{0}(t)} n y_{n}^{n-1}\left(\xi^{\prime}\right) \psi_{n, 2 l}\left(\xi^{\prime}\right)\left(\xi^{\prime}\right)^{-3} d \xi^{\prime} ; \quad l=1 ; 3 ; \\
& C_{6,2 l}=-\int_{0}^{1} P_{6}(t) P_{2 l}(t) d t \int_{\xi_{1}(n)}^{\xi_{0}(t)} n y_{n}^{n-1}\left(\xi^{\prime}\right) \psi_{n, 2 l}\left(\xi^{\prime}\right)\left(\xi^{\prime}\right)^{-5} d \xi^{\prime} ; \quad l=1 ; 2 .
\end{aligned}
$$

The approximation of E. Milne-S. Chandrasekhar $[7,8]$ corresponds to

$$
\tilde{\alpha}_{2} \simeq-\frac{5}{6} \xi_{1}^{2}\left\{3 \psi_{n, 2}\left(\xi_{1}\right)+\xi_{1} \frac{d}{d \xi_{1}} \psi_{n, 2}\left(\xi_{1}\right)\right\}^{-1}
$$

where $\xi_{1} \equiv \xi_{1}(n)$ is the dimensionless radius of Emden's sphere for the polytrope with index $n$. The self-consistent calculation of rotational ellipsoid parameters is implemented in the same way as at $n=1$ using the iterative method. In zero approximation $\alpha_{2}=\tilde{\alpha}_{2}, \alpha_{4}=\alpha_{6}=0$.

\subsection{Polytrope $n=2$}

The solutions of equation (37) calculated by numerical integration can be approximately represented in the form:

$$
\begin{aligned}
\psi_{2,0}(\xi) & =\frac{c_{2} \xi^{2}+c_{4} \xi^{4}+c_{6} \xi^{6}}{1+b_{2} \xi^{2}+b_{4} \xi^{4}+b_{6} \xi^{6}} \\
c_{2} & =0.166966, \quad c_{4}=0.00468588, \quad c_{6}=0.000390954, \\
b_{2} & =0.130027, \quad b_{4}=0.00193647, \quad b_{6}=-3.53099 \cdot 10^{-5} \\
\psi_{2,2}(\xi) & =j_{2}(\sqrt{2} \xi)+\frac{a_{0}+a_{2} \xi^{2}+a_{4} \xi^{4}}{1+d_{2} \xi^{2}+d_{4} \xi^{4}+d_{6} \xi^{6}+d_{8} \xi^{8}} \xi^{4}, \\
a_{0} & =-3.41171, \quad a_{2}=-0.0453981, \quad a_{4}=0.0479275 \\
d_{2} & =47.1876, \quad d_{4}=10.8073, \quad d_{6}=-0.0769964, \quad d_{8}=0.049992 ; \\
\psi_{2,4}(\xi) & =j_{4}(\sqrt{2} \xi)+\frac{g_{0}+g_{2} \xi^{2}+g_{4} \xi^{4}}{1+h_{2} \xi^{2}+h_{4} \xi^{4}} \xi^{6}, \\
g_{0} & =-0.00455907, \quad g_{2}=0.000294272, \quad g_{4}=-2.68804 \cdot 10^{-6} \\
h_{2} & =1.01463, \quad h_{4}=0.0870029 ;
\end{aligned}
$$


Table 3. Dependence of the model characteristics with index $n=2$ on angular velocity.

\begin{tabular}{|c|c|c|c|c|c|c|c|}
\hline$\Omega$ & $e(\Omega)$ & $\xi_{p}(\Omega)$ & $\xi_{e}(\Omega)$ & $\alpha_{2}(\Omega)$ & $\alpha_{4}(\Omega)$ & $\eta(n, \Omega)$ & $\zeta(n, \Omega)$ \\
\hline \hline 0.01000 & 0.04433 & 4.35153 & 4.35581 & -9.64290 & $-2.46122 \cdot 10^{-6}$ & 1.00036 & 1.00110 \\
\hline 0.02000 & 0.08876 & 4.34747 & 4.36470 & -9.66576 & $-3.98549 \cdot 10^{-5}$ & 1.00143 & 1.00443 \\
\hline 0.03000 & 0.13341 & 4.34065 & 4.37980 & -9.70428 & -0.00020592 & 1.00324 & 1.01006 \\
\hline 0.04000 & 0.17840 & 4.33094 & 4.40155 & -9.75915 & -0.000670219 & 1.00580 & 1.01809 \\
\hline 0.05000 & 0.22386 & 4.31822 & 4.43065 & -9.83133 & -0.00170194 & 1.00915 & 1.02869 \\
\hline 0.06000 & 0.26994 & 4.30228 & 4.46812 & -9.92219 & -0.00371262 & 1.01333 & 1.04212 \\
\hline 0.07000 & 0.31687 & 4.28289 & 4.51546 & -10.03360 & -0.00733287 & 1.01842 & 1.05869 \\
\hline 0.08000 & 0.36488 & 4.25973 & 4.57492 & -10.16800 & -0.0135554 & 1.02448 & 1.07888 \\
\hline 0.09000 & 0.41439 & 4.23240 & 4.64998 & -10.32890 & -0.0240197 & 1.03165 & 1.10330 \\
\hline 0.10000 & 0.46602 & 4.20036 & 4.74648 & -10.52110 & -0.0416372 & 1.04008 & 1.13286 \\
\hline 0.11000 & 0.52092 & 4.16271 & 4.87540 & -10.75250 & -0.072176 & 1.05000 & 1.16892 \\
\hline 0.12000 & 0.58171 & 4.11752 & 5.06216 & -11.03780 & -0.129377 & 1.06177 & 1.21369 \\
\hline 0.13000 & 0.65800 & 4.05799 & 5.40334 & -11.42520 & -0.263157 & 1.07605 & 1.27150 \\
\hline 0.13100 & 0.66800 & 4.05034 & 5.46177 & -11.47750 & -0.28819 & 1.07767 & 1.27838 \\
\hline 0.13200 & 0.67916 & 4.04210 & 5.53204 & -11.53650 & -0.318497 & 1.07933 & 1.28557 \\
\hline 0.13300 & 0.69225 & 4.03313 & 5.62230 & -11.60730 & -0.357261 & 1.08106 & 1.29318 \\
\hline 0.13400 & 0.71000 & 4.02345 & 5.75907 & -11.70950 & -0.414263 & 1.08290 & 1.30157 \\
\hline 0.13410 & 0.71243 & 4.02249 & 5.77902 & -11.72450 & -0.422289 & 1.08310 & 1.30251 \\
\hline 0.13420 & 0.71515 & 4.02155 & 5.80160 & -11.74180 & -0.43126 & 1.08330 & 1.30348 \\
\hline 0.13430 & 0.71833 & 4.02068 & 5.82821 & -11.76270 & -0.441664 & 1.08351 & 1.30452 \\
\hline 0.13440 & 0.72241 & 4.01995 & 5.86242 & -11.79080 & -0.454742 & 1.08373 & 1.30569 \\
\hline 0.134494 & 0.73067 & 4.01996 & 5.92954 & -11.85470 & -0.479288 & 1.08402 & 1.30742 \\
\hline
\end{tabular}

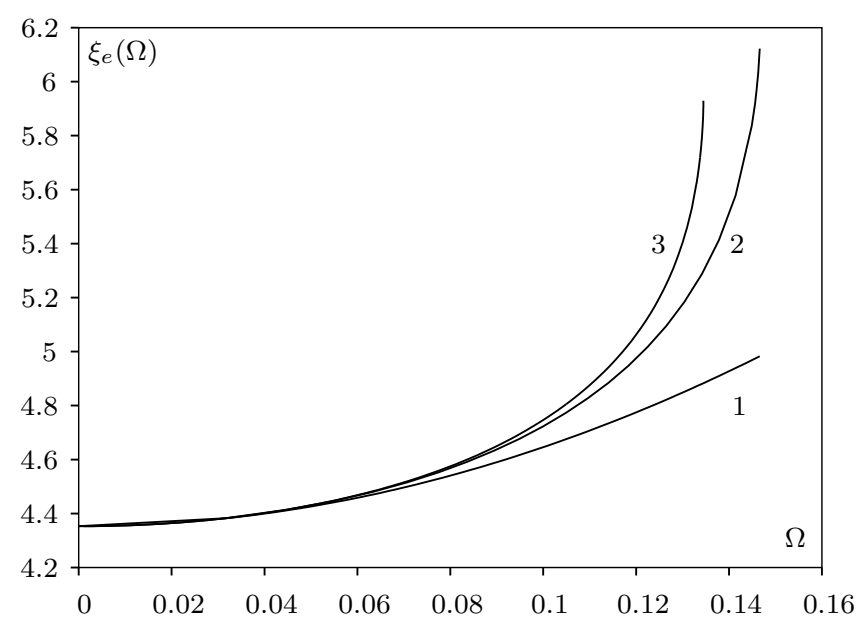

Fig. 9. Dependence of the equatorial radius $\xi_{e}(\Omega)$ on rotation velocity $\Omega$ for the polytrope $n=2$ in different approximations. Curve 1 built on the results of works [7, 8], curve 2 corresponds to the results [9]. Curve 3 built according to the Table 3 .
The equatorial and polar radii, eccentricity, integration constants, relative stellar mass and relative moment of inertia as the functions of angular velocity $\Omega$ (Table 3 ) are found by the method of successive approximations using the solution of equation (7) in the approximation

$Y_{2}(\xi, \theta)=y_{2}(\xi)+\psi_{2,0}(\xi)+\sum_{l=1}^{2} \alpha_{2 l} \psi_{2,2 l}(\xi) P_{2 l}(t)$,

the system of equations (39) in the approximation $\alpha_{2 l}=0$ at $l \geqslant 3$ and the expressions (40). As was shown from the Table the maximal value of angular velocity $\Omega_{\max }(2)=0.13449$. In Fig. 9 was shown calculated by us the dependence of equatorial radius on angular velocity (curve 3) as well as, for the comparison, the results of E. Milne-S. Chandrasekhar calculations $[7,8]$ (curve 1) and R. James [9] (curve 2).

\subsection{Polytrope $n=3$}

In this case the asymptotic region of the function $\psi_{3,2 l}(\xi)$ (where they are close to the Bessel function) is small $(\xi<1)$, therefore for the convenience we represent these functions in the form of Pade approximant:

$$
\psi_{3,0}(\xi)=\frac{c_{2} \xi^{2}+c_{4} \xi^{4}+c_{6} \xi^{6}}{1+b_{2} \xi^{2}+b_{4} \xi^{4}+b_{6} \xi^{6}},
$$




$$
\begin{aligned}
c_{2} & =0.175687, \quad c_{4}=0.0248748, \quad c_{6}=0.00071618, \\
b_{2} & =0.353168, \quad b_{4}=0.00229006, \quad b_{6}=1.20061 \cdot 10^{-5} ; \\
\psi_{3,2}(\xi)= & \frac{a_{2} \xi^{2}+a_{4} \xi^{4}+a_{6} \xi^{6}}{1+d_{2} \xi^{2}+d_{4} \xi^{4}}, \\
a_{2} & =0.061913, \quad a_{4}=0.00289971, \quad a_{6}=0.000346914 \\
d_{2} & =0.192777, \quad d_{4}=0.0229925 ; \\
\psi_{3,4}(\xi)= & \frac{g_{4} \xi^{4}+g_{6} \xi^{6}}{1+h_{2} \xi^{2}+h_{4} \xi^{4}}, \\
g_{4} & =0.00581656, \quad g_{6}=0.00533426 \\
h_{2} & =10.6029, \quad h_{4}=0.00160348 .
\end{aligned}
$$

Analogue of the function (42) is the function

$$
Y_{3}(\xi, \theta)=y_{3}(\xi)+\psi_{3,0}(\xi)+\sum_{l=1}^{2} \alpha_{2 l} \psi_{3,2 l}(\xi) P_{2 l}(t) .
$$

The integration constants $\alpha_{2 l}$ are determined self-consistently in the same way as at $n=2$. The results of calculation of geometrical and physical characteristics as well as the integration constants are shown in Table 4. The dependencies of equatorial and polar radii in different approximations are given in Figs. 10, 11. In the Fig. 10, curve 1 corresponds to approximation of E. Milne-S. Chandrasekhar, curve built on the results R. James's work and curve 3 - according to Table 4 . In the Fig. 11, curve 1 corresponds to approximation of E. Milne-S. Chandrasekhar, which almost coincides with the R. James's results. Curve 3 built on the results of Table 4 .

Table 4. Dependence of the model characteristics with index $n=3$ on angular velocity.

\begin{tabular}{|c|c|c|c|c|c|c|c|}
\hline$\Omega$ & $e(\Omega)$ & $\xi_{p}(\Omega)$ & $\xi_{e}(\Omega)$ & $\alpha_{2}(\Omega)$ & $\alpha_{4}(\Omega)$ & $\eta(n, \Omega)$ & $\zeta(n, \Omega)$ \\
\hline \hline 0.00500 & 0.04576 & 6.89548 & 6.90271 & -10.85850 & $6.10036 \cdot 10^{-10}$ & 1.00015 & 1.00060 \\
\hline 0.01000 & 0.09174 & 6.89132 & 6.92051 & -10.88610 & $3.99801 \cdot 10^{-8}$ & 1.00061 & 1.00242 \\
\hline 0.01500 & 0.13818 & 6.88425 & 6.95093 & -10.93320 & $4.74182 \cdot 10^{-7}$ & 1.00139 & 1.00549 \\
\hline 0.02000 & 0.18533 & 6.87403 & 6.99520 & -11.00150 & $2.82479 \cdot 10^{-6}$ & 1.00248 & 1.00985 \\
\hline 0.02500 & 0.23350 & 6.86031 & 7.05531 & -11.09370 & $1.16593 \cdot 10^{-5}$ & 1.00389 & 1.01559 \\
\hline 0.03000 & 0.28308 & 6.84260 & 7.13431 & -11.21420 & $3.85724 \cdot 10^{-5}$ & 1.00564 & 1.02282 \\
\hline 0.03500 & 0.33458 & 6.82022 & 7.23700 & -11.36920 & 0.000110925 & 1.00776 & 1.03169 \\
\hline 0.04000 & 0.38875 & 6.79216 & 7.37127 & -11.56930 & 0.000292576 & 1.01025 & 1.04243 \\
\hline 0.04500 & 0.44684 & 6.75683 & 7.55127 & -11.83270 & 0.000739428 & 1.01315 & 1.05537 \\
\hline 0.05000 & 0.51124 & 6.71119 & 7.80647 & -12.19610 & 0.00188011 & 1.01652 & 1.07105 \\
\hline 0.05500 & 0.58822 & 6.64689 & 8.21991 & -12.75880 & 0.00525887 & 1.02043 & 1.09055 \\
\hline 0.05600 & 0.60665 & 6.62964 & 8.34315 & -12.92000 & 0.0066597 & 1.02130 & 1.09511 \\
\hline 0.05700 & 0.62701 & 6.60958 & 8.49371 & -13.11250 & 0.00861298 & 1.02220 & 1.09999 \\
\hline 0.05800 & 0.65044 & 6.58515 & 8.68921 & -13.35530 & 0.0115234 & 1.02314 & 1.10529 \\
\hline 0.05900 & 0.67988 & 6.55253 & 8.97737 & -13.69790 & 0.0164819 & 1.02413 & 1.11127 \\
\hline 0.05910 & 0.68345 & 6.54848 & 9.01616 & -13.74260 & 0.0172007 & 1.02424 & 1.11192 \\
\hline 0.05920 & 0.68721 & 6.54421 & 9.05802 & -13.79050 & 0.0179878 & 1.02434 & 1.11260 \\
\hline 0.05930 & 0.69120 & 6.53968 & 9.10357 & -13.84230 & 0.0188571 & 1.02445 & 1.11329 \\
\hline 0.05940 & 0.69546 & 6.53484 & 9.15370 & -13.89870 & 0.0198282 & 1.02456 & 1.11401 \\
\hline 0.05950 & 0.70008 & 6.52964 & 9.20970 & -13.96120 & 0.0209287 & 1.02467 & 1.11476 \\
\hline 0.05960 & 0.70518 & 6.52396 & 9.27356 & -14.03160 & 0.0222023 & 1.02478 & 1.11555 \\
\hline 0.05970 & 0.71095 & 6.51767 & 9.34874 & -14.11360 & 0.023723 & 1.02490 & 1.11639 \\
\hline 0.05980 & 0.71782 & 6.51047 & 9.44222 & -14.21410 & 0.0256391 & 1.02502 & 1.11731 \\
\hline 0.05990 & 0.72696 & 6.50163 & 9.57350 & -14.35310 & 0.0283622 & 1.02515 & 1.11840 \\
\hline 0.05998 & 0.74248 & 6.48989 & 9.81476 & -14.60370 & 0.0333914 & 1.02530 & 1.11987 \\
\hline
\end{tabular}




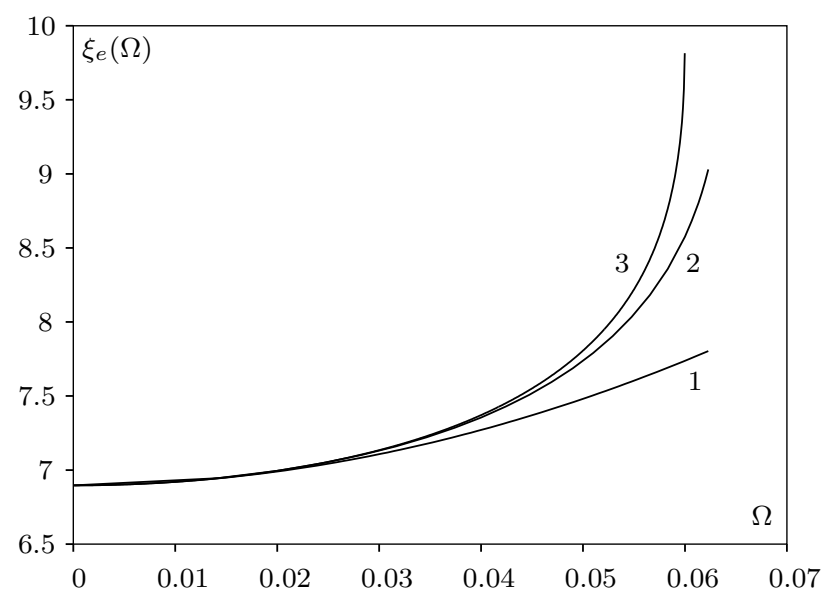

Fig. 10. Dependence of the equatorial radius $\xi_{e}(\Omega)$ on rotation velocity $\Omega$ for the polytrope $n=3$ in different approximations. Curve 1 built on the results of works $[7,8]$, curve 2 corresponds to the results [9]. Curve 3 built according to the Table 4.

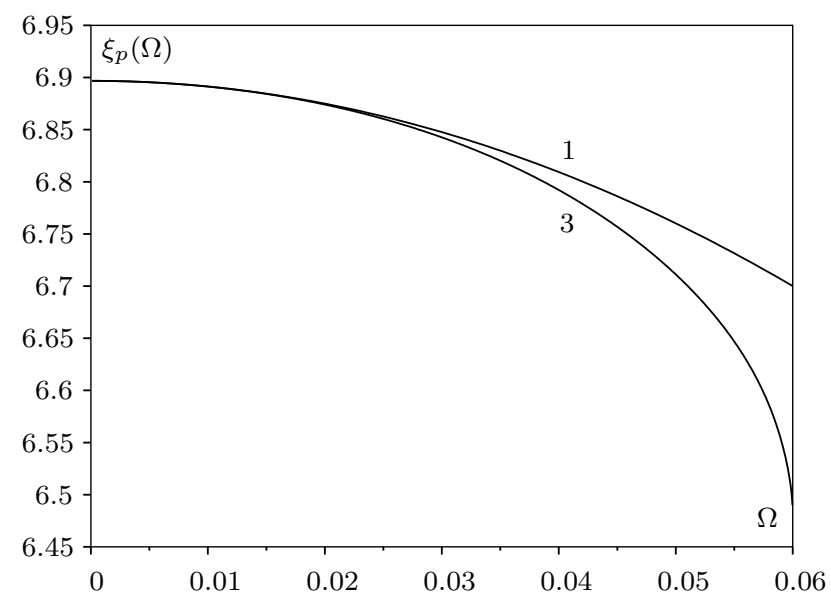

Fig. 11. Dependence of the polar radius $\xi_{p}(\Omega)$ on rotation velocity $\Omega$ for the polytrope $n=3$ in different approximations. Curve 1 built on the results of works $[7,8]$, curve 3 built according to the Table 4 .

\section{Conclusions}

Simultaneous employment of the differential and integral form of mechanical equilibrium equation yields a closed system of expressions to determine the geometrical and physical characteristics of polytropic star with axial rotation.

Model with index $n=1$ allows to write the analytical solution of two-dimensional differential mechanical equilibrium equation in the form of expansion of Legendre polynomials and first order Bessel spherical functions, in which appears the set of integration constants $A_{2 l}$ (f. (26)). Use of the integral equilibrium equation (27) fully illustrates the benefits of the proposed method, because it gives an opportunity to gain the system of linear algebraic equations for finding constants of integration, therefore there is the possibility of self-consistent calculation of the geometrical ellipsoid characteristics, as well as establishment of stability range of the stars regarding to the rotation velocity. The precision of calculations of $\Omega_{\max }$, like all other characteristics of the star, depends on the number of terms considered in the series (26) and on precision of matrix elements calculation (30). As was shown from the Figs. 6, 7, the approximation of E. Milne-S. Chandrasekhar $[7,8]$ (only term of type $A_{2} j_{2}(\xi) P_{2}(t)$ is taken into account and $S_{2,2}$ is determined by the formula (31)) satisfactorily describes variation of polar and equatorial radii only in range of small angular velocities $\left(\Omega \leqslant 0.5 \Omega_{\max }\right)$. Good convergence of series (26) provides high precision of calculation of model characteristics in all region of angular velocity variations $0 \leqslant \Omega \leqslant \Omega_{\max }$.

Table 5. Dependence of maximal angular velocity on polytropic index in different approximations.

\begin{tabular}{|c|c|c|c|c|}
\hline$n$ & 0 & 1 & 2 & 3 \\
\hline \hline$\Omega_{\max }^{C h}(n)$ & - & 0.3315 & 0.1805 & 0.0793 \\
\hline$\Omega_{\max }^{J}(n)$ & - & 0.29 & 0.147 & 0.062 \\
\hline$\Omega_{\max }^{*}(n)$ & 0.47399 & 0.24607 & 0.13449 & 0.05998 \\
\hline
\end{tabular}

Dependence of maximal angular velocity on polytropic index in different approximations is shown in Table $5, \Omega_{\max }^{C h}(n)$ corresponds to the approximation of E. Milne-S. Chandrasekhar's $[7,8], \Omega_{\max }^{J}(n)$ - the R. James's results [9], $\Omega_{\text {max }}^{*}(n)$ - corresponds to our results according to the Tables $2-4$. As was shown from the Table 5, the maximal value of angular rotation velocity decreases with the increasing $n$ proportional to $\Omega_{\max }(n) \approx 2^{1-n} \Omega_{\max }(1)$. Therefore in the case $n>1$ it becomes possible to 
approximate the description of equilibrium by linearization of the equation (7). In this case the convergence of series for the functions $\psi_{n, 2 l}(\xi) P_{2 l}(t)$ improves with increasing of polytropic index as evidenced by results for the models with $n=2$ and 3, shown in Tables 3 and 4 .

Geometrical and physical characteristics for the polytropic models are monotonic functions of polytropic index $n$, dimensionless angular velocity $\Omega$ as well as the constants $K$ and $\rho_{c}$. Below are given the general expressions for the equatorial $R_{e}\left(n, \Omega \mid K, \rho_{c}\right)$ and polar $R_{p}\left(n, \Omega \mid K, \rho_{c}\right)$ radii, total mass $M\left(n, \Omega \mid K, \rho_{c}\right)$, volume $V(n, \Omega \mid K)$ and moment of inertia relative to rotational axis $I\left(n, \Omega \mid K, \rho_{c}\right)$ :

$$
\begin{aligned}
R_{e}\left(n, \Omega \mid K, \rho_{c}\right) & =x \rho_{c}^{-\frac{1}{2}(1-1 / n)} \xi_{e}(n, \Omega) ; \\
R_{p}\left(n, \Omega \mid K, \rho_{c}\right) & =x \rho_{c}^{-\frac{1}{2}(1-1 / n)} \xi_{p}(n, \Omega) ; \\
M\left(n, \Omega \mid K, \rho_{c}\right) & =4 \pi x^{3} \rho_{c}^{-\frac{1}{2}(1-3 / n)} \beta_{2}(n) \eta(n, \Omega) ; \\
V(n, \Omega \mid K) & =\frac{4}{3} \pi x^{3} \xi_{e}^{2}(n, \Omega) \xi_{p}(n, \Omega) ; \\
I\left(n, \Omega \mid K, \rho_{c}\right) & =\frac{8}{3} \pi x^{5} \rho_{c}^{-\frac{3}{2}(1-5 / 6 n)} \beta_{4}(n) \eta(n, \Omega) ; \\
x & \equiv\{K(1+n) / 4 \pi G\}^{1 / 2} .
\end{aligned}
$$

[1] Lane H. J. On the Theoretical Temperature of the Sun under the Hypothesis of a gaseous mass Maintining Its Volume by Its Internal Heat and Depending on the Law of Gases Known to Terrestrial Experiment. Am. J. Sci. 50, 57-74 (1870).

[2] Emden K. Gaskugeln: Anwendungen der mechanischen Wärmetheorie. Leipzig, Berlin (1907).

[3] Fowler R. H. Emden's equation: The solutions of Emden's and similar differential equations. Monthly Notices of the Royal Astronomical Society. 91 (1), 63-91 (1930).

[4] Ritter A. Untersuchungen über die Höhe der Atmosphäre und die Constitution gasförmiger Weltkörper. Ann. Phys. 241, 543-558 (1878).

[5] Kelvin W. Mathematical and Physical Papers. 3, 255 (1911).

[6] Eddington A. S. The Internal Constitution of the Stars. Cambridge, Cambridge University Press (1926).

[7] Milne E. A. The equilibrium of a rotating star. Monthly Notices of the Royal Astronomical Society. 83, 118-147 (1923).

[8] Chandrasekhar S. The Equilibrium of Distorted Polytropes. I. The Rotational Problem. Monthly Notices of the Royal Astronomical Society. 33, 390-406 (1933).

[9] James R. A. The Structure and Stability of Rotating Cas Masses. Astrophysical Journal. 140, 552-582 (1964).

[10] Vavrukh M. V., Smerechynskyi S. V., Tyshko N. L. The microscopic parameters and the macroscopic characteristics of real degenerate dwarfs. Journal of Physical Studies. 14 (4), 4901 (2010).

[11] Shapiro S. L., Teukolsky S. A. Black Holes, White Dwarfs and Neutron Stars. Cornell University, Ithaca, New York (1983).

[12] Chandrasekhar S. An Introduction to the Study of Stellar Structure. Chicago, University of Chicago Press (1939).

[13] Lyttleton R. A. The Stability of Rotating Liquid Masses. Cambridge, Cambridge University Press (1953).

[14] Abramowitz M., Stegun I. A. Handbook of Mathematical Functions With Formulas, Graphs, and Mathematical Tables. Government Printing Office Washington (1972). 


\title{
Самоузгоджений опис рівноваги зір з осьовим обертанням
}

\author{
Ваврух М. В., Тишко Н. Л., Дзіковський Д. В., Стельмах О. М. \\ Львівсъкий національний університет імені Івана Франка, \\ вул. Кирила і Мефодія, 8, Лъвів, 79005, Україна
}

\begin{abstract}
Запропоновано новий підхід до опису механічної рівноваги зір з осьовим обертанням. Самоузгоджений розрахунок грунтується на одночасному використанні диференціальної та інтегральної форм рівняння рівноваги, що дає змогу коректно визначати сталі інтегрування. У межах політропної моделі з індексами $n=0$ та 1 вперше одержано аналітичні розв'язки, для $n=2$ і 3 - числові. Розраховано геометричні параметри поверхні зорі, її масу, об'єм та момент інерції як функції кутової швидкості. Знайдено максимальні значення кутової швидкості, за яких порушується стабільність зір. Одержані результати уточнюють результати Е. Мілна, С. Чандрасекара та Р. Джеймса, одержані за допомогою наближеного чисельного інтегрування рівняння рівноваги.
\end{abstract}

Ключові слова: зорі-політропи, неоднорідні еліпсоїди, осъове обертання, рівняння механічної рівноваги, стабільність зір.

2000 MSC: $85-08,85 \mathrm{~A} 15$

УдК: 524.3 